\title{
Exact Magnetothermoelastic Solution for a Hollow Sphere Subjected to Initial Stress, Rotation, and Magnetic Field
}

\author{
S. M. Abo-Dahab, ${ }^{1,2}$ Nahed S. Hussein,, ${ }^{2,3}$ and M. Al-Bokamy ${ }^{2}$ \\ ${ }^{1}$ Mathematics Department, Faculty of Science, SVU, Qena 83523, Egypt \\ ${ }^{2}$ Mathematics Department, Faculty of Science, Taif University, P.O. Box 888, Altaif 21944, Saudi Arabia \\ ${ }^{3}$ Mathematics Department, Faculty of Science, Cario University, Giza 12613, Egypt
}

Correspondence should be addressed to Nahed S. Hussein; hussein.nahed@yahoo.com

Received 18 December 2013; Accepted 14 February 2014; Published 14 April 2014

Academic Editor: Bo Yu

Copyright (c) 2014 S. M. Abo-Dahab et al. This is an open access article distributed under the Creative Commons Attribution License, which permits unrestricted use, distribution, and reproduction in any medium, provided the original work is properly cited.

We estimated an analytical solution of the displacement, stress, and temperature in a rotating isotropic homogeneous elastic medium hollow sphere subjected to periodic loading and magnetic field. The coupled theory of thermoelasticity is applied to determine an infinite velocity of heat propagation. The numerical calculations are carried out for the displacement, temperature, and stresses. The results obtained are displayed graphically to illustrate the effect of initial stress, rotation, and magnetic field which indicate to pronounce influence of rotation and magnetic field.

\section{Introduction}

The classical and generalized theories of coupled thermoelasticity are extensively developed due to their many applications in the advanced structural design problems. Therefore, it is crucial to obtain the deformation and temperature distributions in the structures under thermal shock loads. In the classical coupled problems of thermoelasticity, the first time rate of change in the first invariant of the strain tensor appears in the first law of thermodynamics, causing coupling between the elastic and thermal fields. The mathematical treatment of coupled thermoelasticity problems by analytical methods is rather complicated. In basic problems of the coupled thermoelasticity theory, such as the problems of infinite and half spaces, analytical treatment can be found in the literature. Also, there are a large number of articles, in which numerical methods are used. Dynamical problems of thermodiffusion in solids are discussed by Nowacki ([13]). Lord and Shulman [4] introduced the first generalized dynamical theory of thermoelasticity to calculate an infinite speed for the waves due to the thermal field. The generalized thermoelasticity for an isotropic media is illustrated by Dhaliwal and Sherief [5]. Abd-Alla and Mahmoud [6] studied the problem of magnetothermoelastic in rotating nonhomogeneous orthotropic hollow cylinder under the hyperbolic heat conduction model. Abd-Alla et al. [7] investigated thermal stresses in a nonhomogeneous orthotropic elastic multilayered cylinder. A generalized magnetothermoelasticity in a perfectly conducting medium is discussed by Ezzat and Youssef [8]. Othman [9] studied effect of rotation and relaxation time on thermal shock problem for a half-space in generalized thermoviscoelasticity. Othman [10] investigated Lord-Shulman theory under the dependence of the modulus of elasticity on the reference temperature in two-dimensional generalized thermoelasticity. Green and Lindsay [11] developed the theory of thermoelasticity using two thermal relaxation times to measure the origin of the infinite speed.

Magnetothermoelasticity has a lot of applications in diverse fields as geophysics, engineering, acoustics, and other fields. Effect of rotation on plane waves of the generalized magnetothermoelasticity or electromagnetothermoviscoelasticity with two relaxation times or rotation is pointed out by Othman and Song $[12,13]$. Sherief et al. [14] investigated theory of generalized thermoelastic diffusion. Sherief 
and Saleh [15] discussed half-space problem in the theory of generalized thermoelastic diffusion. Reflection of SV waves from the free surface of an elastic solid in generalized thermoelastic diffusion has been obtained by Singh [16]. Kumar and Kansal [17] discussed propagation of Lamb waves in transversely isotropic thermoelastic diffusive plate. Thermomechanical response of generalized thermoelastic diffusion with one relaxation time due to time harmonic sources was discussed by Ram et al. [18]. Aouadi [19] examined the thermoelastic diffusion problem for an infinite elastic body with spherical cavity. Abo-Dahab and Singh [20] investigated the effect of magnetic field on wave propagation in generalized thermoelastic solid with diffusion. Othman et al. [21] discussed the effect of diffusion in a two-dimensional problem of generalized thermoelasticity with Green-Naghdi theory. Xia et al. [22] studied the influence of diffusion on generalized thermoelastic problems of infinite body with a cylindrical cavity. Deswal and Kalkal [23] studied the twodimensional generalized electromagnetothermoviscoelastic problem for a half-space with diffusion. Abd-Alla and AboDahab [24] found the time-harmonic sources in a generalized magnetothermoviscoelastic continuum with and without energy dissipation. Abo-Dahab and Mohamed [25] discussed the effect of magnetic field and hydrostatic initial stress on reflection phenomena of $\mathrm{P}$ and $\mathrm{SV}$ waves from a generalized thermoelastic solid half-space. Abd-Alla et al. [26] studied the generalized magnetothermoelastic Rayleigh waves in a granular medium under the influence of a gravity field and initial stress. Roychoudhuri and Mukhopadhyay [27] discussed effect of rotation and relaxation times on plane waves in generalized thermoviscoelasticity. Recently, some new works in isotropic or orthotropic elastic media have been discussed in [28-31].

The present investigation is devoted to study the interaction between the processes of elasticity, rotation, magnetic field, initial stress, heat, and diffusion in an infinite elastic solid with a spherical cavity in the context of the coupled thermoelastic diffusion. The temperature, displacements, stresses, diffusion concentration, and chemical potential are obtained in the physical domain using the harmonic vibrations. The problem of coupled thermoelasticity has been reduced as a special case. The variations of rotation, magnetic field on the stresses, displacements, and temperature distribution have been studied and shown graphically.

\section{Mathematical Formulation of the Problem}

Let us consider a perfect electric conductor and linearized Maxwell equation governing the electromagnetic field in absence of the displacement current (SI) as in Roychoudhuri and Mukhopadhyay [27] and Kraus [32]:

$$
\begin{gathered}
\vec{J}=\operatorname{Curl} \vec{h}, \\
\operatorname{Curl} \vec{E}=-\mu_{e} \frac{\partial \vec{h}}{\partial t}, \\
\operatorname{div} \vec{h}=0,
\end{gathered}
$$

$$
\begin{gathered}
\operatorname{div} \vec{E}=0, \\
\vec{h}=\operatorname{Curl}(\vec{u} \wedge \vec{H}), \\
\vec{E}=-\mu_{e}\left(\frac{\partial \vec{u}}{\partial t} \wedge \vec{H}\right),
\end{gathered}
$$

where $\vec{u}$ is the component of displacement vector, $\vec{h}$ the perturbed magnetic field vector, $\vec{J}$ the electric current density vector, $\vec{E}$ the electric intensity vector, and $\mu_{e}$ the magnetic permeability. Applying an initial magnetic field vector $\vec{H}=$ $\left(0,0, H_{\phi}\right)$ in spherical coordinates $(r, \theta, \phi)$ to (1) we have

$$
\begin{gathered}
\vec{u}=u(r, t) \underline{e_{r}}=(u(r, t), 0,0), \\
\vec{H}=\left(0,0, H_{\phi}\right), \\
\vec{E}=-\mu_{e}\left(0,-H_{\phi} \frac{\partial u}{\partial t}, 0\right), \\
\vec{J}=\left(0, \frac{-\partial h_{\phi}}{\partial r}, 0\right), \\
h_{\phi}=-H_{\phi}\left(\frac{\partial u}{\partial r}+\frac{2 u}{r}\right), \\
\vec{E}=\left(0, E_{2}, 0\right) .
\end{gathered}
$$

The elastic medium is rotating uniformly with the angular velocity $\underline{\Omega}=\Omega \underline{n}$, where $\underline{n}$ is a unit vector representing the direction of the axis of rotation. The displacement equation of motion in the rotation frame has two additional terms $\vec{\Omega} \wedge(\vec{\Omega} \wedge \vec{u})$ which is the centripetal acceleration due to timevarying motion only and $2 \vec{\Omega} \wedge \overrightarrow{\dot{u}}$ is the Coriolis acceleration which neglected, and $\vec{\Omega}=(0, \Omega, 0)$.

The governing equation for an isotropic, homogeneous elastic solid with generalized magnetothermoelastic under effect of rotation is as follows.

(i) Equation of motion:

$$
\frac{\partial \sigma_{r r}}{\partial r}+\frac{2}{r}\left(\sigma_{r r}-\sigma_{\theta \theta}\right)+F_{r}=\rho\left(\frac{\partial^{2} u}{\partial t^{2}}-\Omega^{2} u\right) ;
$$

we know that

$$
\begin{aligned}
\vec{\Omega} \wedge\left(\vec{\Omega} \wedge u \underline{e}_{r}\right) & =\vec{\Omega} \wedge\left(\vec{\Omega} \underline{e}_{\theta} \wedge u \underline{e}_{r}\right) \\
& =\Omega \underline{e}_{\theta} \wedge\left(-\Omega u \underline{e}_{\phi}\right)=-\Omega^{2} u \underline{e}_{r},
\end{aligned}
$$

where $F_{r}$ is defined as the radial component of Lorentz's force, which may be written as

$$
\begin{aligned}
\vec{F}=\mu_{e}(\vec{J} \wedge \vec{H}) & =\left(\mu_{e} H_{\phi}^{2} \frac{\partial}{\partial r}\left(\frac{\partial u}{\partial r}+\frac{2 u}{r}\right), 0,0\right) \\
& =\mu_{e} H_{0}^{2}\left(\frac{\partial}{\partial r}\left(\frac{\partial u}{\partial r}+\frac{2 u}{r}\right), 0,0\right) .
\end{aligned}
$$


(ii) Equation of heat conduction:

$$
\begin{gathered}
K \nabla^{2} T=\rho c_{\gamma} \frac{\partial T}{\partial t}+\gamma T_{0}\left(\frac{\partial \dot{u}}{\partial r}+\frac{2 \dot{u}}{r}\right), \\
K\left[\frac{\partial^{2} T}{\partial r^{2}}+\frac{2}{r} \frac{\partial T}{\partial r}\right]=\rho c_{v} \frac{\partial T}{\partial t}+\gamma T_{0}\left(\frac{\partial \dot{u}}{\partial r}+\frac{2 \dot{u}}{r}\right),
\end{gathered}
$$

where $c_{v}$ is the specific heat per unit mass, $K$ is the thermal conductivity, $T$ is the absolute temperature, $T_{0}$ is the reference temperature, $\rho$ is the density, $\gamma=\alpha_{c}(3 \lambda+2 \mu)$, and $\alpha_{c}$ is the thermal expansion.

For a spherical symmetric the nonvanishing components may be written as

$$
\begin{aligned}
\sigma_{r r} & =2 \mu \frac{\partial u}{\partial r}+\lambda\left(\frac{\partial u}{\partial r}+\frac{2 u}{r}\right)-(3 \lambda+2 \mu) \alpha_{c} T \\
\sigma_{\phi \phi} & =\sigma_{\theta \theta}=2 \mu \frac{u}{r}+\lambda\left(\frac{\partial u}{\partial r}+\frac{2 u}{r}\right)-(3 \lambda+2 \mu) \alpha_{c} T, \\
\tau_{r r} & =\mu_{e} H_{\phi}^{2}\left(\frac{\partial u}{\partial r}+\frac{2 u}{r}\right)
\end{aligned}
$$

where $\sigma_{i j}(i, j=1,2,3)$ are the stress components tensor and $\lambda$ and $\mu$ are Lame's constants.

Assume that the magnetic permeability $\mu_{e}$ of the isotropic hollow sphere equals the magnetic permeability of the medium around it.

\section{Boundary Conditions}

The homogeneous initial conditions are supplemented by the following boundary conditions:

$$
\begin{aligned}
T & =0 \quad \text { at } r=a, \\
T & =T_{0} \quad \text { at } r=b, \\
\sigma_{r r}+\tau_{r r} & =-P_{0} e^{i w t} \quad \text { at } r=b, \\
\sigma_{r r}+\tau_{r r} & =0 \quad \text { at } r=a .
\end{aligned}
$$

\section{Solution of the Problem}

Taking the harmonic vibrations we assume

$$
\begin{aligned}
& u(r, t)=u^{\prime}(r) e^{i w t}, \\
& T(r, t)=T^{\prime}(r) e^{i w t},
\end{aligned}
$$

where $\omega$ is the natural frequency of the vibration.
By using (7) and substituting into (3) and (6) then

$$
\begin{gathered}
\frac{\partial}{\partial r}\left[(2 \mu+\lambda) \frac{\partial u}{\partial r}+\frac{2 \lambda u}{r}-(3 \lambda+2 \mu) \alpha_{c} T\right] \\
+\frac{2}{r}\left(2 \mu \frac{\partial u}{\partial r}-2 \mu \frac{u}{r}\right) \\
+\mu_{e} H_{\phi}^{2} \frac{\partial}{\partial r}\left(\frac{\partial u}{\partial r}+\frac{2 u}{r}\right)+\rho \Omega^{2} u=\rho \frac{\partial^{2} u}{\partial t^{2}} \\
(2 \mu+\lambda) \frac{\partial^{2} u}{\partial r^{2}}+\frac{2(2 \mu+\lambda)}{r} \frac{\partial u}{\partial r}-\frac{2(2 \mu+\lambda)}{r^{2}} u \\
+\mu_{e} H_{\phi}^{2}\left[\frac{\partial^{2} u}{\partial r^{2}}+\frac{2}{r} \frac{\partial u}{\partial r}-\frac{2 u}{r^{2}}\right] \\
\quad(3 \lambda+2 \mu) \alpha_{c} \frac{\partial T}{\partial r}+\rho \Omega^{2} u=\rho \frac{\partial^{2} u}{\partial t^{2}}, \\
\left(2 \mu+\lambda+\mu_{e} H_{\phi}^{2}\right)\left[\frac{\partial^{2} u}{\partial r^{2}}+\frac{2}{r} \frac{\partial u}{\partial r}-\frac{2 u}{r^{2}}\right]-\alpha_{c}(3 \lambda+2 \mu) \frac{\partial T}{\partial r} \\
=\frac{\partial^{2} T}{\partial r^{2}}-\rho \Omega^{2} u \\
\left.\frac{\partial}{\partial r}\right]=\rho c_{\gamma} \frac{\partial T}{\partial t}+\gamma T_{0} \frac{\partial}{\partial t}\left(\frac{\partial u}{\partial r}+\frac{2 u}{r}\right) ;
\end{gathered}
$$

by using

$$
\begin{aligned}
& u(r, t)=u^{\prime}(r) e^{i w t}, \\
& T(r, t)=T^{\prime}(r) e^{i w t}
\end{aligned}
$$

we get

$$
\begin{aligned}
&(2 \mu\left.+\lambda+\mu_{e} H_{\varphi}^{2}\right)\left[\frac{d^{2} u^{\prime}}{d r^{2}}+\frac{2}{r} \frac{d u^{\prime}}{d r}-\frac{2 u^{\prime}}{r^{2}}\right] \\
&-(3 \lambda+2 \mu) \frac{d T^{\prime}}{d r}=-\left(\omega^{2} \rho+\rho \Omega^{2}\right) u^{\prime} \\
&=-\rho\left(\omega^{2}+\Omega^{2}\right) u^{\prime}(r), \\
& K\left[\frac{d^{2} T^{\prime}}{d r^{2}}+\frac{2}{r} \frac{d T^{\prime}}{d r}\right]=i \rho c_{\gamma} \omega T^{\prime}+i \gamma T_{0} \omega\left(\frac{d u^{\prime}}{d r}+\frac{2 u^{\prime}}{r}\right) .
\end{aligned}
$$

Using the potential function $\phi^{*}$ then we assume $\vec{u} \cdot \vec{r}=d \phi^{*} / d r$ and (12) and (13) may be transformed to

$$
\begin{array}{r}
\left(2 \mu+\lambda+\mu_{e} H_{\phi}^{2}\right)\left[\frac{d^{3} \phi^{*}}{d r^{3}}+\frac{2}{r} \frac{d^{2} \phi^{*}}{d r^{2}}-\frac{2}{r^{2}} \frac{d \phi^{*}}{d r}\right] \\
-(3 \lambda+2 \mu) \frac{d T^{\prime}}{d r}=-\rho\left(\omega^{2}+\Omega^{2}\right) \frac{d \phi^{*}}{d r},
\end{array}
$$




$$
\begin{aligned}
(2 \mu & \left.+\lambda+\mu_{e} H_{\phi}^{2}\right) \frac{d}{d r}\left[\frac{d^{2} \phi^{*}}{d r^{2}}+\frac{2}{r} \frac{d \phi^{*}}{d r}\right] \\
& -(3 \lambda+2 \mu) \frac{d T^{\prime}}{d r}=-\rho\left(\omega^{2}+\Omega^{2}\right) \frac{d \phi^{*}}{d r}
\end{aligned}
$$

by integrating respect to $r$

$$
\begin{aligned}
& \left(2 \mu+\lambda+\mu_{e} H_{\phi}^{2}\right)\left[\frac{d^{2} \phi^{*}}{d r^{2}}+\frac{2}{r} \frac{d \phi^{*}}{d r}\right]-(3 \lambda+2 \mu) T^{\prime} \\
& =-\rho\left(\omega^{2}+\Omega^{2}\right) \phi^{*} \\
& \left(2 \mu+\lambda+\mu_{e} H_{\phi}^{2}\right) \nabla^{2} \phi^{*}-(3 \lambda+2 \mu) T^{\prime} \\
& =-\rho\left(\omega^{2}+\Omega^{2}\right) \phi^{*} \\
& {\left[\left(2 \mu+\lambda+\mu_{e} H_{\phi}^{2}\right) \nabla^{2}+\rho\left(\omega^{2}+\Omega^{2}\right)\right] \phi^{*}} \\
& =(3 \lambda+2 \mu) T^{\prime} .
\end{aligned}
$$

From (13), we obtain

$$
\begin{aligned}
& K \nabla^{2} T^{\prime}-i \rho c_{\gamma} \omega T^{\prime} \\
&= \frac{i \gamma T_{0} \omega}{\left(2 \mu+\lambda+\mu_{e} H_{\phi}^{2}\right)}\left[(3 \lambda+2 \mu) T^{\prime}-\rho\left(\omega^{2}+\Omega^{2}\right)\right] \phi^{*} \\
& K \nabla^{2} T^{\prime}-\left(i \rho c_{\gamma} \omega T^{\prime}+\frac{i \gamma T_{0} \omega(3 \lambda+2 \mu)}{\left(2 \mu+\lambda+\mu_{e} H_{\phi}^{2}\right)}\right) T^{\prime} \\
&=\frac{-i \gamma T_{0} \omega \rho\left(\omega^{2}+\Omega^{2}\right) \phi^{*}}{\left(2 \mu+\lambda+\mu_{e} H_{\phi}^{2}\right)} .
\end{aligned}
$$

Equation (15) can be written as

$$
\begin{gathered}
{\left[\nabla^{2}+\frac{\rho\left(\omega^{2}+\Omega^{2}\right)}{\left(2 \mu+\lambda+\mu_{e} H_{\phi}^{2}\right)}\right] \phi^{*}} \\
=\frac{(3 \lambda+2 \mu)}{\left(2 \mu+\lambda+\mu_{e} H_{\phi}^{2}\right)} T^{\prime} .
\end{gathered}
$$

Equation (16) takes the form

$$
\begin{aligned}
{\left[\nabla^{2}\right.} & \left.+\left(\frac{i \rho c_{\gamma} \omega}{K}+\frac{i \gamma T_{0} \omega(3 \lambda+2 \mu)}{\left(2 \mu+\lambda+\mu_{e} H_{\phi}^{2}\right)}\right)\right] T^{\prime} \\
& =\frac{-i \gamma T_{0} \omega \rho\left(\omega^{2}+\Omega^{2}\right) \phi^{*}}{\left(2 \mu+\lambda+\mu_{e} H_{\phi}^{2}\right)}
\end{aligned}
$$

which can be written also as

$$
\begin{aligned}
& \left(\nabla^{2}+\alpha_{1}^{2}\right) \phi^{*}=\eta_{1} T^{\prime}, \\
& \left(\nabla^{2}+\beta^{2}\right) T^{\prime}=\eta_{2} \phi^{*},
\end{aligned}
$$

where

$$
\begin{aligned}
& \alpha_{1}^{2}=\frac{\rho\left(\omega^{2}+\Omega^{2}\right)}{\left(2 \mu+\lambda+\mu_{e} H_{\phi}^{2}\right)}, \\
& \eta_{1}=\frac{(3 \lambda+2 \mu)}{\left(2 \mu+\lambda+\mu_{e} H_{\phi}^{2}\right)}, \\
& \beta^{2}=-i\left(\frac{\rho c_{\gamma} \omega}{K}+\frac{\gamma T_{0} \omega(3 \lambda+2 \mu)}{\left(2 \mu+\lambda+\mu_{e} H_{\phi}^{2}\right)}\right) .
\end{aligned}
$$

Decoupling (19) one may obtain

$$
\left(\nabla^{2}+m_{1}^{2}\right)\left(\nabla^{2}+m_{2}^{2}\right)\left(\phi^{*}, T^{\prime}\right)=0
$$

where $m_{1}$ and $m_{2}$ are roots with positive real parts of biquadratic equation:

$$
m^{4}+\left(\alpha_{1}^{2}+\beta^{2}-\eta_{1} \eta_{2}\right) m^{2}+\alpha_{1}^{2} \beta^{2}=0 .
$$

Assuming the regularity conditions for $\dot{T}$ and $\phi^{*}$, the solutions of (22) are obtained in terms of spherical Hankel's function in the form

$$
\begin{gathered}
\phi^{*}(r)=A_{1} h_{0}^{(2)}\left(m_{1} r\right)+A_{2} h_{0}^{(2)}\left(m_{2} r\right), \\
T^{\prime}(r)=A_{3} h_{0}^{(2)}\left(m_{1} r\right)+A_{4} h_{0}^{(2)}\left(m_{2} r\right),
\end{gathered}
$$

where $A_{1}, A_{2}, A_{3}$, and $A_{4}$ are constants.

Consider

$$
\begin{gathered}
u^{\prime}(r)=\frac{d \phi^{*}(r)}{d r}, \\
u^{\prime}(r)=A_{1} h_{1}^{(2)}\left(m_{1} r\right)+A_{2} h_{1}^{(2)}\left(m_{2} r\right), \\
u(r, t)=\left[A_{1} h_{1}^{(2)}\left(m_{1} r\right)+A_{2} h_{1}^{(2)}\left(m_{2} r\right)\right] e^{i w t}, \\
T(r, t)=\left[A_{3} h_{0}^{(2)}\left(m_{1} r\right)+A_{4} h_{0}^{(2)}\left(m_{2} r\right)\right] e^{i w t}, \\
\sigma_{r r}=2 \mu \frac{\partial u}{\partial r}+\lambda\left(\frac{\partial u}{\partial r}+\frac{2 u}{r}\right)-(3 \lambda+2 \mu) \alpha_{c} T \\
\sigma_{r r}=\left(2 \mu \left[\frac{A_{1}}{2}\left(h_{0}^{(2)}\left(m_{1} r\right)-h_{2}^{(2)}\left(m_{1} r\right)\right)\right.\right. \\
\left.+\frac{A_{2}}{2}\left(h_{0}^{(2)}\left(m_{2} r\right)-h_{2}^{(2)}\left(m_{2} r\right)\right)\right] \\
+\lambda\left[\frac{A_{1}}{2}\left(h_{0}^{(2)}\left(m_{1} r\right)-h_{2}^{(2)}\left(m_{1} r\right)\right)\right. \\
\left.+\frac{A_{2}}{2}\left(h_{0}^{(2)}\left(m_{2} r\right)-h_{2}^{(2)}\left(m_{2} r\right)\right)\right] \\
+\frac{2 \lambda}{r}\left[A_{1} h_{1}^{(2)}\left(m_{1} r\right)+A_{2} h_{1}^{(2)}\left(m_{2} r\right)\right] \\
\left.-(3 \lambda+2 \mu) \alpha_{c}\left[A_{3} h_{0}^{(2)}\left(m_{1} r\right)+A_{4} h_{0}^{(2)}\left(m_{2} r\right)\right]\right) e^{i w t},
\end{gathered}
$$




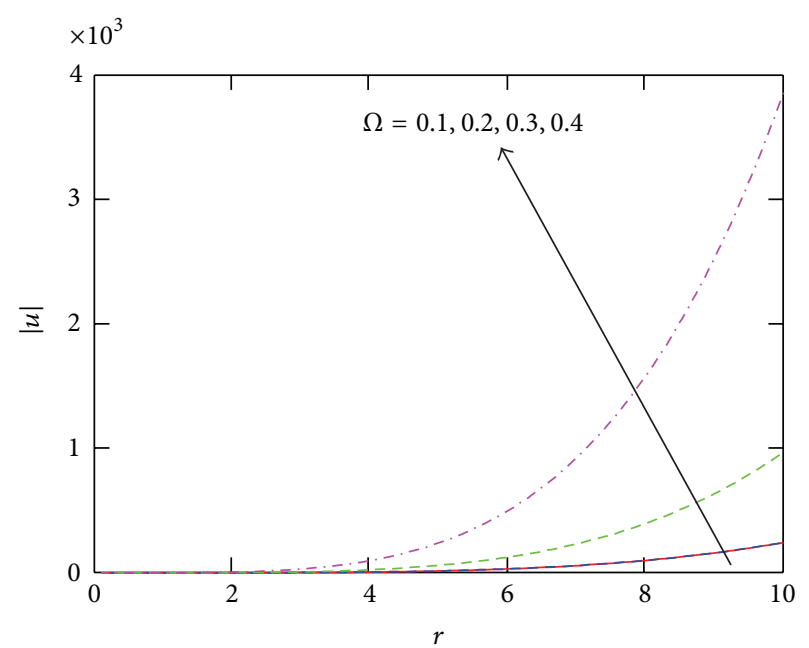

(a)

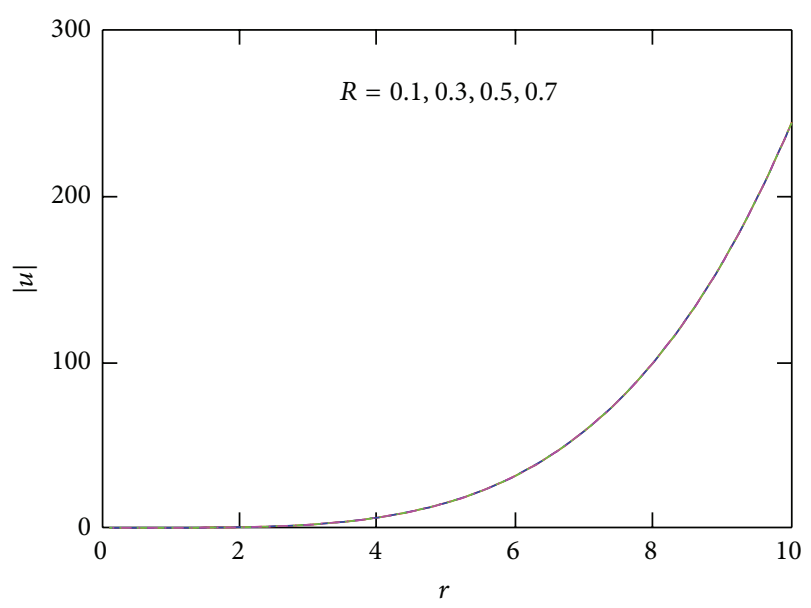

(c)

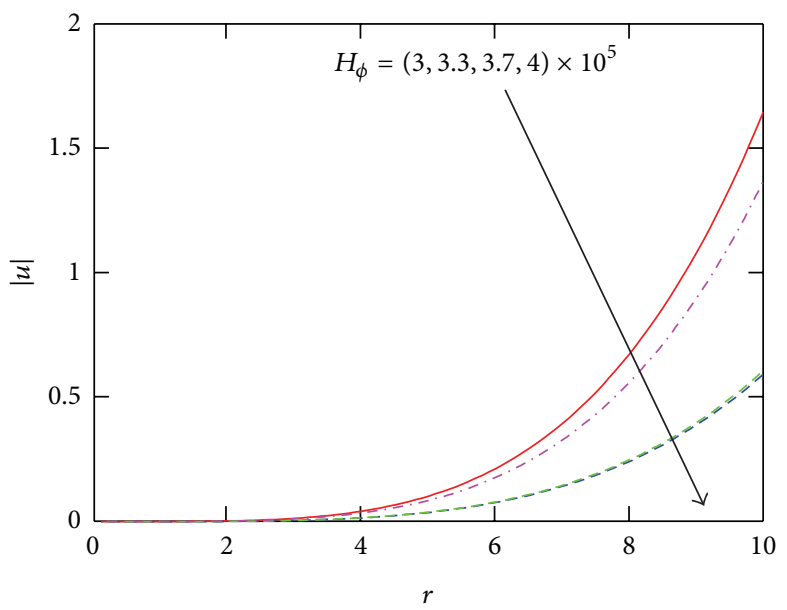

(b)

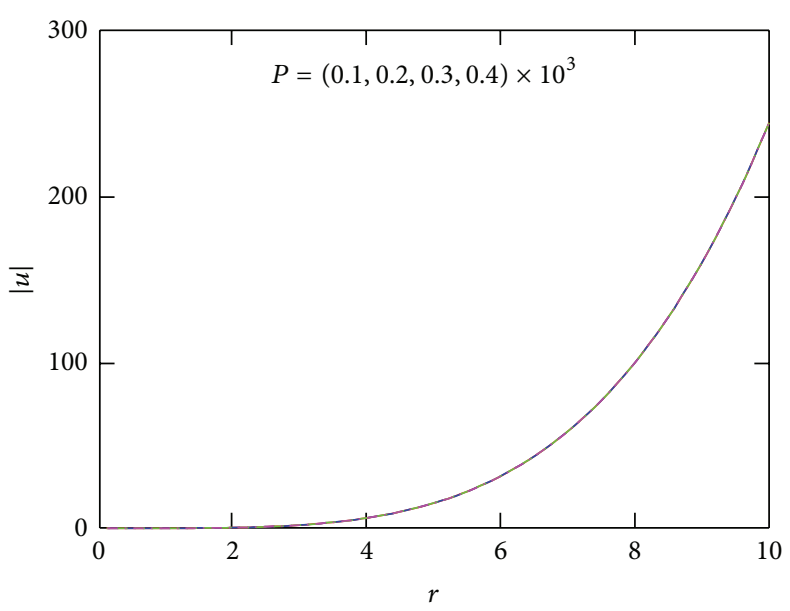

(d)

FIgURE 1: Variation of the absolute values of the displacement $u$ with varies values of $\Omega, H_{\phi}, R$, and $P$ with respect to the radius $r$.

which tends to

$$
\begin{gathered}
\sigma_{r r}=\left[\left[\left(\mu+\frac{\lambda}{2}\right) h_{0}^{(2)}\left(m_{1} r\right)-\left(\mu+\frac{\lambda}{2}\right) h_{2}^{(2)}\left(m_{1} r\right)\right] A_{1}\right. \\
+\left(\mu+\frac{\lambda}{2}\right)\left[h_{0}^{(2)}\left(m_{2} r\right)-h_{2}^{(2)}\left(m_{2} r\right)\right] A_{2} \\
+\frac{2 \lambda}{r} h_{1}^{(2)}\left(m_{1} r\right) A_{1}+\frac{2 \lambda}{r} h_{1}^{(2)}\left(m_{2} r\right) A_{2} \\
\left.-(3 \lambda+2 \mu) \alpha_{c}\left[A_{3} h_{0}^{(2)}\left(m_{1} r\right)+A_{4} h_{0}^{(2)}\left(m_{2} r\right)\right]\right] e^{i w t}, \\
\tau_{r r}=\mu_{e} H_{\phi}^{2}\left(\frac{\partial u}{\partial r}+\frac{2 u}{r}\right), \\
\tau_{r r}=\mu_{e} H_{\phi}^{2}\left[\left(\frac{1}{2}\left[h_{0}^{(2)}\left(m_{1} r\right)-h_{2}^{(2)}\left(m_{1} r\right)\right]\right.\right. \\
\left.+\frac{2}{r} h_{1}^{(2)}\left(m_{1} r\right)\right) A_{1}
\end{gathered}
$$

$$
\begin{array}{r}
+\frac{1}{2}\left[\left(h_{0}^{(2)}\left(m_{2} r\right)-h_{2}^{(2)}\left(m_{2} r\right)\right)\right. \\
\left.\left.+\frac{2}{r} h_{1}^{(2)}\left(m_{2} r\right)\right] A_{2}\right] e^{i w t}
\end{array}
$$$$
\sigma_{\phi \phi}=\sigma_{\theta \theta}=2 \mu \frac{u}{r}+\lambda\left(\frac{\partial u}{\partial r}+\frac{2 u}{r}\right)-(3 \lambda+2 \mu) \alpha_{c} T,
$$$$
\sigma_{\phi \phi}=\sigma_{\theta \theta}
$$$$
=\left(\frac{2}{r}(\lambda+\mu)\left[A_{1} h_{1}^{(2)}\left(m_{1} r\right)+A_{2} h_{1}^{(2)}\left(m_{2} r\right)\right]\right.
$$$$
+\lambda\left[\frac{A_{1}}{2}\left(h_{0}^{(2)}\left(m_{1} r\right)-h_{2}^{(2)}\left(m_{1} r\right)\right)\right.
$$$$
\left.+\frac{A_{2}}{2}\left(h_{0}^{(2)}\left(m_{2} r\right)-h_{2}^{(2)}\left(m_{2} r\right)\right)\right]
$$$$
\left.-(3 \lambda+2 \mu) \alpha_{c}\left[A_{3} h_{0}^{(2)}\left(m_{1} r\right)+A_{4} h_{0}^{(2)}\left(m_{2} r\right)\right]\right)
$$$$
\times e^{i w t}
$$ 


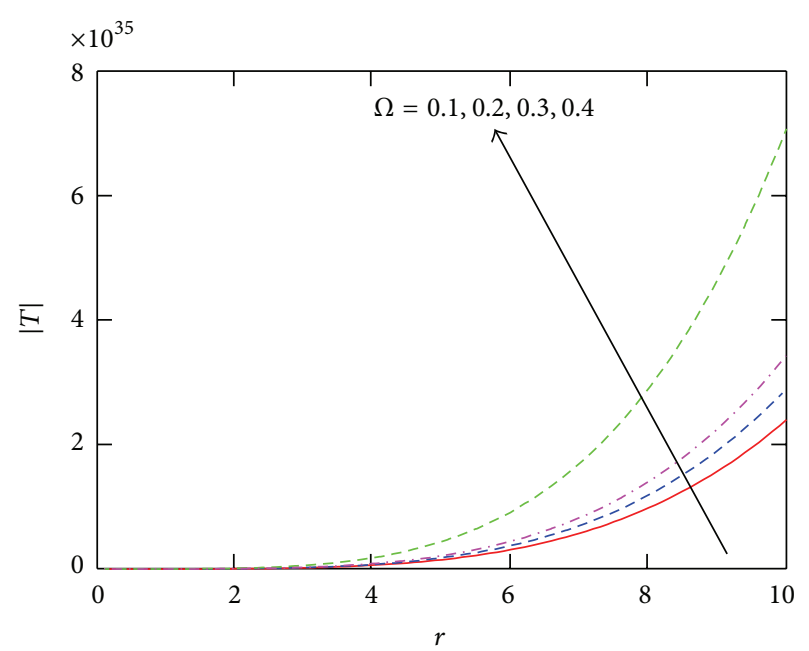

(a)

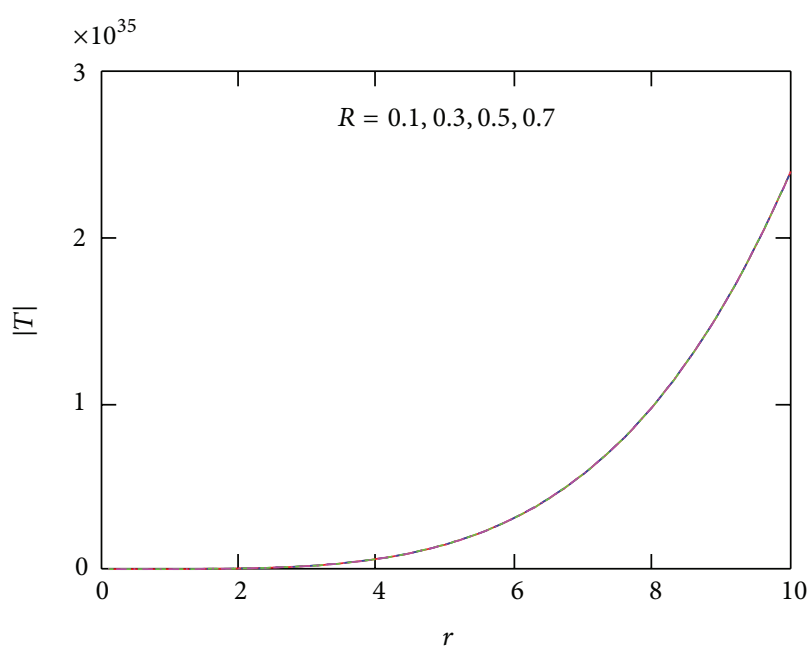

(c)

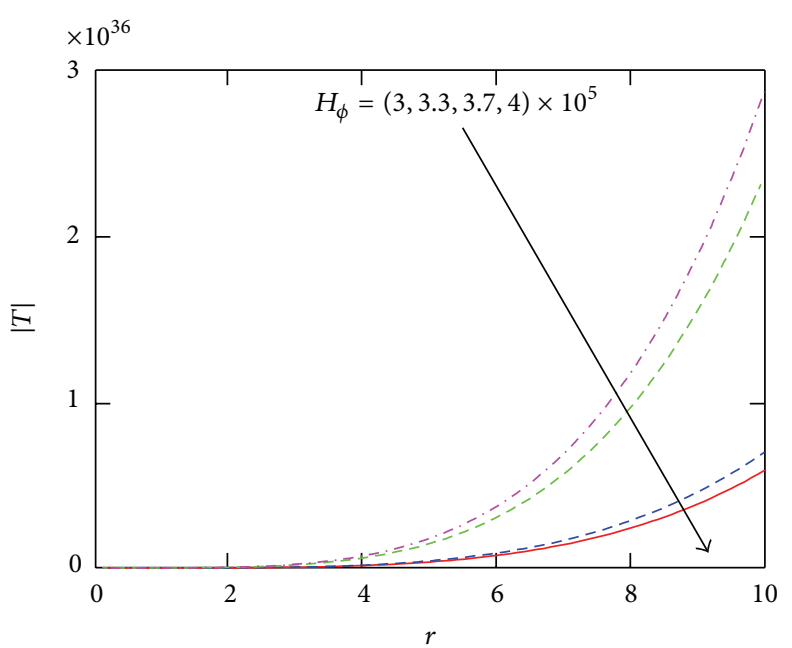

(b)

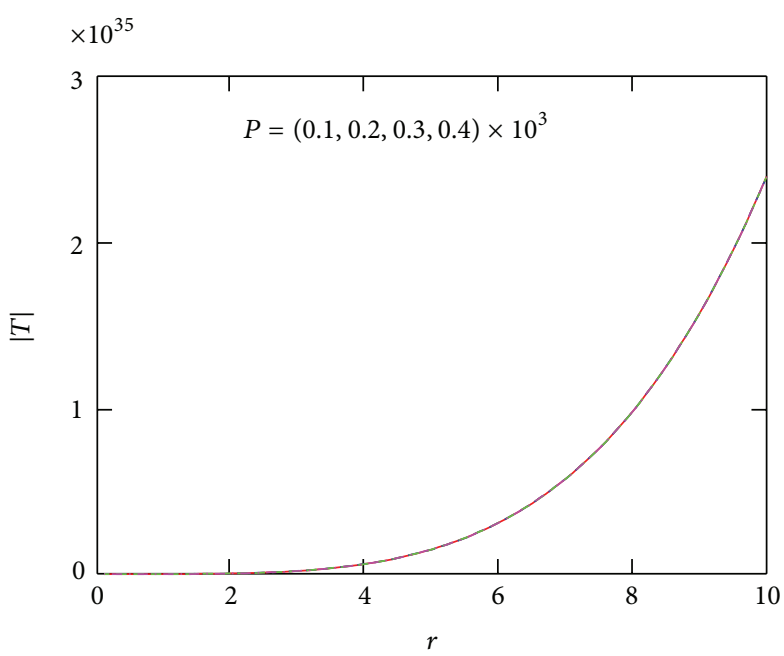

(d)

FIGURE 2: Variation of the absolute values of the temperature $T$ with varies values of $\Omega, H_{\phi}, R$, and $P$ with respect to the radius $r$.

\section{Boundary Conditions}

Consider

$$
\begin{gathered}
T=0 \quad \text { at } r=a, \\
T=T_{0} \quad \text { at } r=b, \\
{\left[A_{3} h_{0}^{(2)}\left(m_{1} a\right)+A_{4} h_{0}^{(2)}\left(m_{2} a\right)\right] e^{i w t}=0,} \\
{\left[A_{3} h_{0}^{(2)}\left(m_{1} b\right)+A_{4} h_{0}^{(2)}\left(m_{2} b\right)\right] e^{i w t}=T_{0},} \\
\sigma_{r r}+\tau_{r r}=0 \quad \text { at } r=a, \\
{\left[\left(\mu+\frac{\lambda}{2}+\frac{\mu_{e} H_{\phi}^{2}}{2}\right) h_{0}^{(2)}\left(m_{1} a\right)\right.} \\
-\left(\mu+\frac{\lambda}{2}+\frac{\mu_{e} H_{\phi}^{2}}{2}\right) h_{2}^{(2)}\left(m_{1} a\right) \\
\left.+\left(\frac{\mu_{e} H_{\phi}^{2}}{a}+\frac{2 \lambda}{a}\right) h_{1}^{(2)}\left(m_{1} a\right)\right] A_{1}
\end{gathered}
$$

$$
\begin{gathered}
+\left[\left(\mu+\frac{\lambda}{2}+\frac{\mu_{e} H_{\phi}^{2}}{2}\right) h_{0}^{(2)}\left(m_{2} a\right)\right. \\
-\left(\mu+\frac{\lambda}{2}+\frac{\mu_{e} H_{\phi}^{2}}{2}\right) h_{2}^{(2)}\left(m_{2} a\right) \\
\left.+\left(\frac{\mu_{e} H_{\phi}^{2}}{a}+\frac{2 \lambda}{a}\right) h_{1}^{(2)}\left(m_{2} a\right)\right] A_{2} \\
-(3 \lambda+2 \mu) \alpha_{c}\left[A_{3} h_{0}^{(2)}\left(m_{1} a\right)+A_{4} h_{0}^{(2)}\left(m_{2} a\right)\right]=0, \\
\sigma_{r r}+\tau_{r r}=-P_{0} e^{i w t} \text { at } r=b, \\
{\left[\left(\mu+\frac{\lambda}{2}+\frac{\mu_{e} H_{\phi}^{2}}{2}\right) h_{0}^{(2)}\left(m_{1} b\right)\right.} \\
-\left(\mu+\frac{\lambda}{2}+\frac{\mu_{e} H_{\phi}^{2}}{2}\right) h_{2}^{(2)}\left(m_{1} b\right)
\end{gathered}
$$




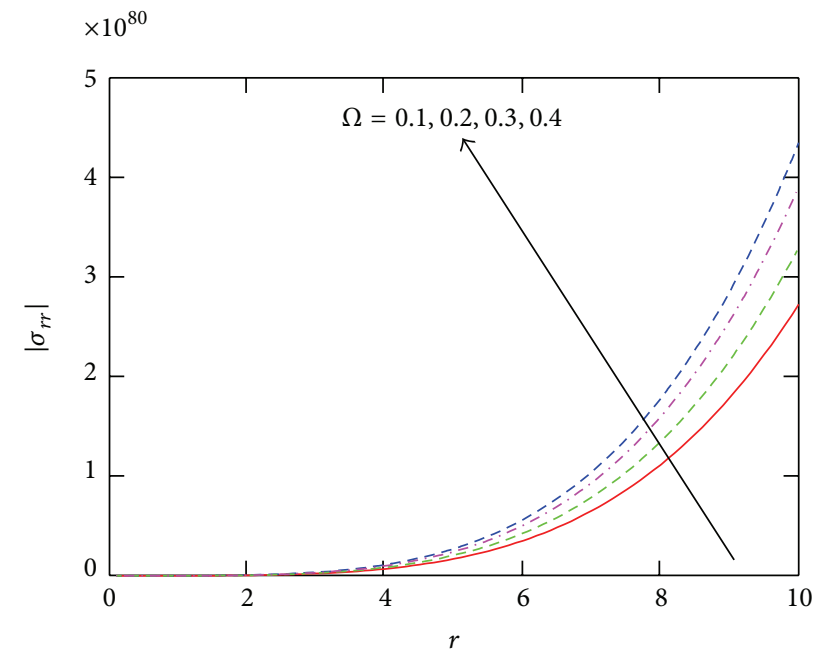

(a)

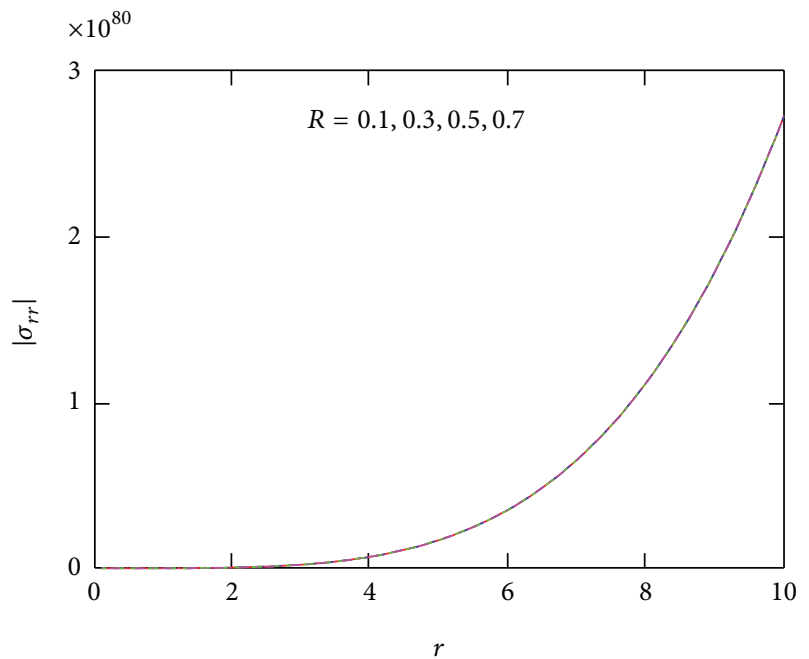

(c)

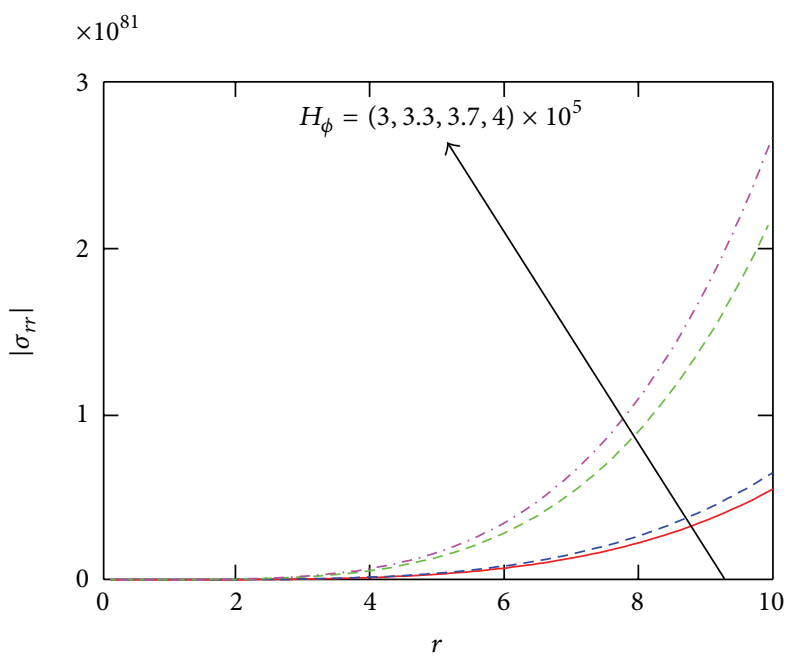

(b)

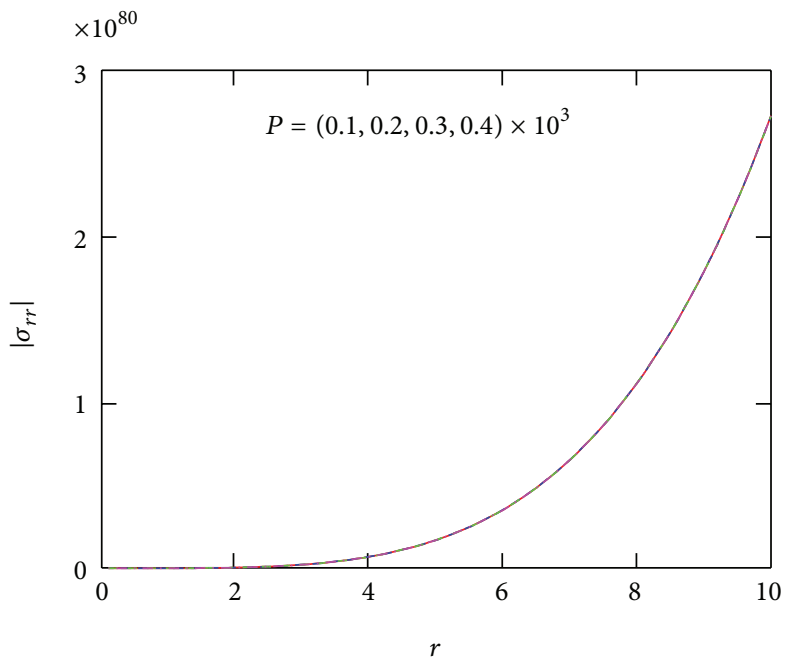

(d)

FIGURE 3: Variation of the absolute values of the radial stress $\sigma_{r r}$ with varies values of $\Omega, H_{\phi}, R$, and $P$ with respect to the radius $r$.

$$
\begin{aligned}
& \left.+\left(\frac{\mu_{e} H_{\phi}^{2}}{b}+\frac{2 \lambda}{b}\right) h_{1}^{(2)}\left(m_{1} b\right)\right] A_{1} \\
& +\left[\left(\mu+\frac{\lambda}{2}+\frac{\mu_{e} H_{\phi}^{2}}{2}\right) h_{0}^{(2)}\left(m_{2} b\right)\right. \\
& +\left(\mu+\frac{\lambda}{2}+\frac{\mu_{e} H_{\phi}^{2}}{2}\right) h_{2}^{(2)}\left(m_{2} b\right) \\
& \left.+\left(\frac{\mu_{e} H_{\phi}^{2}}{b}+\frac{2 \lambda}{b}\right) h_{1}^{(2)}\left(m_{2} b\right)\right] A_{2} \\
& -(3 \lambda+2 \mu) \alpha_{c}\left[A_{3} h_{0}^{(2)}\left(m_{1} b\right)+A_{4} h_{0}^{(2)}\left(m_{2} b\right)\right]=-P_{0},
\end{aligned}
$$

where $P_{0}$ is the initial stress.
Solution of (28) takes the forms

$$
\begin{array}{ll}
A_{1}=\frac{\Delta_{1}}{\Delta}, & A_{2}=\frac{\Delta_{2}}{\Delta} \\
A_{3}=\frac{\Delta_{3}}{\Delta}, & A_{4}=\frac{\Delta_{4}}{\Delta},
\end{array}
$$

where

$$
\begin{aligned}
& \Delta_{1}=2 b h_{0}^{(2)}\left(m_{1} b\right) h_{0}^{(2)}\left(m_{2} a\right) P_{0} \\
& \times\left(4 h_{1}^{(2)}\left(m_{2} a\right)\left(\lambda+\mu_{e} H_{\varphi}^{2}\right)\right. \\
& \quad+b h_{0}^{(2)}\left(m_{2} a\right)\left(\lambda+2 \mu+\mu_{e} H_{\varphi}^{2}\right) \\
&\left.\quad-b h_{2}^{(2)}\left(m_{2} a\right)\left(\lambda+2 \mu+\mu_{e} H_{\phi}^{2}\right)\right)
\end{aligned}
$$




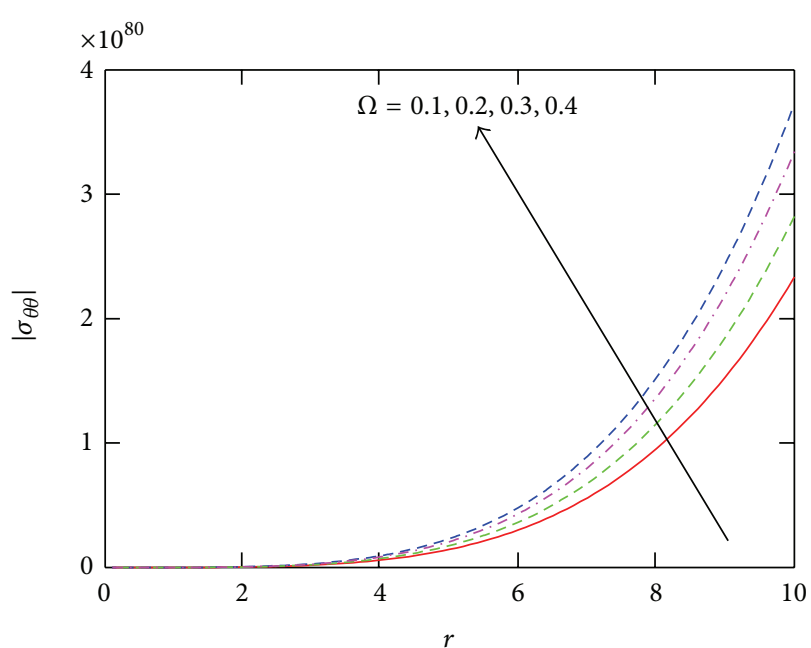

(a)

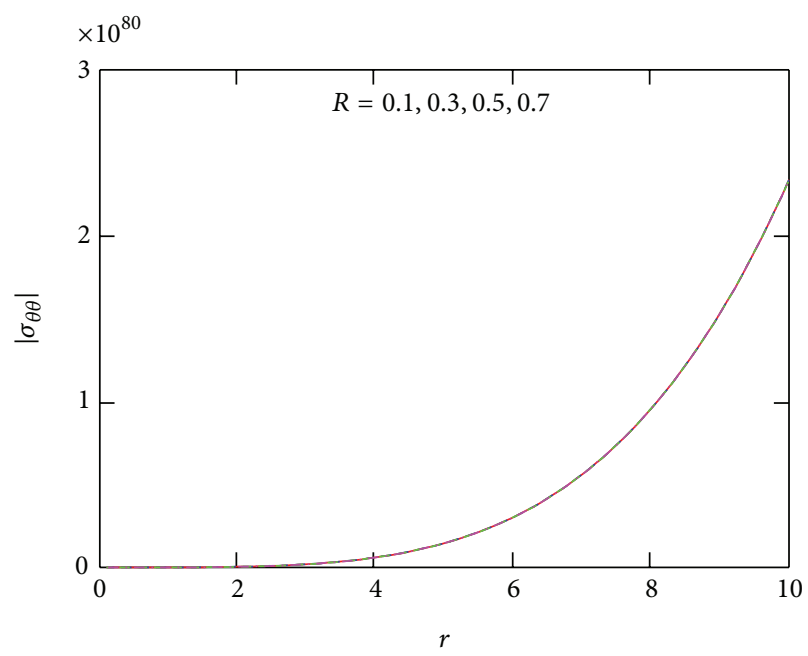

(c)

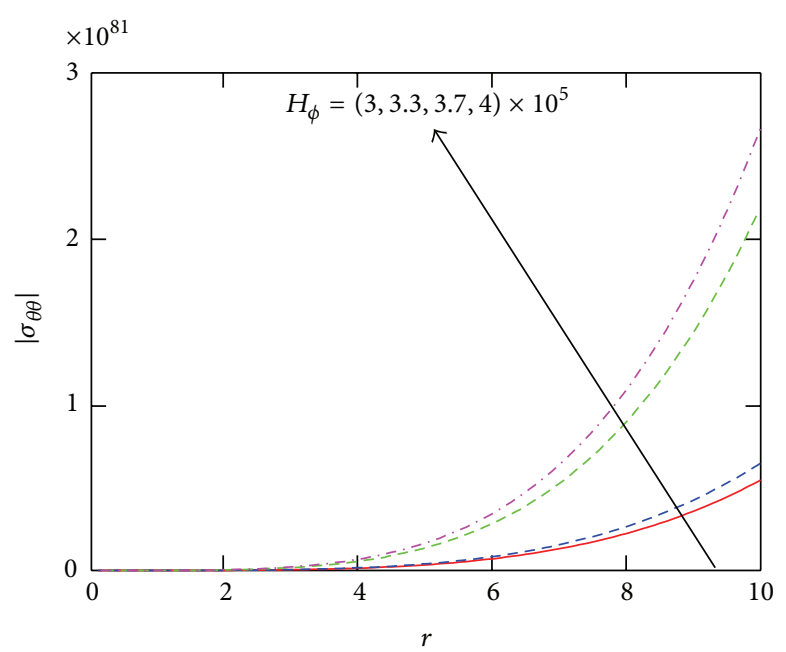

(b)

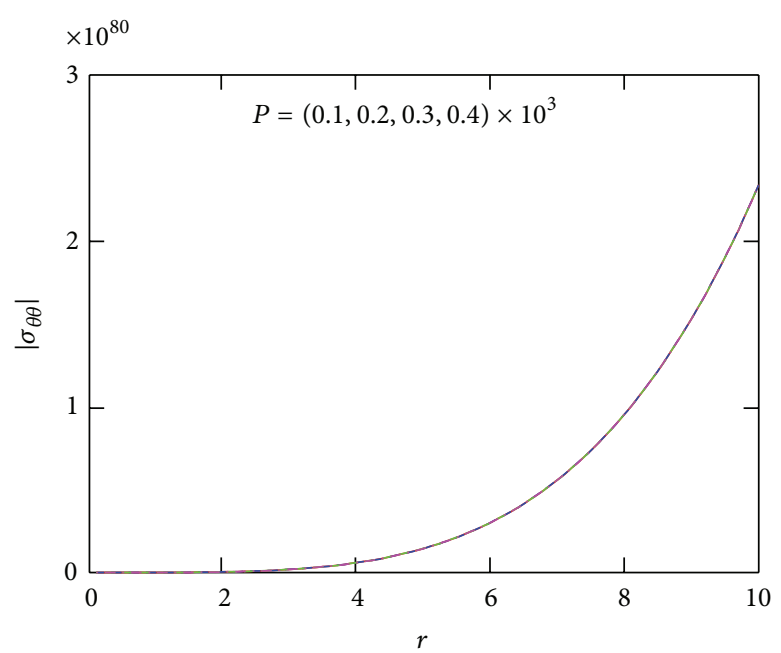

(d)

FIGURE 4: Variation of the absolute values of the hoop stress $\sigma_{r r}$ with varies values of $\Omega, H_{\phi}, R$, and $P$ with respect to the radius $r$.

$$
\begin{aligned}
& -h_{0}^{(2)}\left(m_{1} a\right) h_{0}^{(2)}\left(m_{2} b\right) P_{0} \\
& \times\left(4 h_{1}^{(2)}\left(m_{2} a\right)\left(\lambda+\mu_{e} H_{\phi}^{2}\right)\right. \\
& +b h_{0}^{(2)}\left(m_{2} a\right)\left(\lambda+2 \mu+\mu_{e} H_{\phi}^{2}\right) \\
& \left.\quad-b h_{2}^{(2)}\left(m_{2} a\right)\left(\lambda+2 \mu+\mu_{e} H_{\phi}^{2}\right)\right) \\
& +(3 \lambda+2 \mu) h_{0}^{(2)}\left(m_{1} a\right) h_{0}^{(2)}\left(m_{1} b\right) T_{0} \alpha_{c} \\
& \times\left(4 h_{1}^{(2)}\left(m_{2} a\right)\left(\lambda+\mu_{e} H_{\phi}^{2}\right)\right. \\
& +b h_{0}^{(2)}\left(m_{2} a\right)\left(\lambda+2 \mu+\mu_{e} H_{\phi}^{2}\right) \\
& -b h_{2}^{(2)}\left(m_{2} a\right)\left(\lambda+2 \mu+\mu_{e} H_{\phi}^{2}\right) \\
& -(3 \lambda+2 \mu) h_{0}^{(2)}\left(m_{1} b\right) h_{0}^{(2)}\left(m_{2} a\right) T_{0} \alpha_{c}
\end{aligned}
$$

$$
\begin{aligned}
\times( & 4 h_{1}^{(2)}\left(m_{2} a\right)\left(\lambda+\mu_{e} H_{\phi}^{2}\right) \\
+ & b h_{0}^{(2)}\left(m_{2} a\right)\left(\lambda+2 \mu+\mu_{e} H_{\phi}^{2}\right) \\
- & b h_{2}^{(2)}\left(m_{2} a\right)\left(\lambda+2 \mu+\mu_{e} H_{\phi}^{2}\right) \\
- & (3 \lambda+2 \mu) h_{0}^{(2)}\left(m_{1} a\right)^{2} T_{0} \alpha_{c} \\
\times & \left(4 h_{1}^{(2)}\left(m_{2} b\right)\left(\lambda+\mu_{e} H_{\phi}^{2}\right)\right. \\
& +b h_{0}^{(2)}\left(m_{2} b\right)\left(\lambda+2 \mu+\mu_{e} H_{\phi}^{2}\right) \\
& \left.-b h_{2}^{(2)}\left(m_{2} b\right)\left(\lambda+2 \mu+\mu_{e} H_{\phi}^{2}\right)\right) \\
+ & (3 \lambda+2 \mu) h_{0}^{(2)}\left(m_{1} a\right) \\
\times & h_{0}^{(2)}\left(m_{2} a\right) T_{0} \alpha_{c}
\end{aligned}
$$




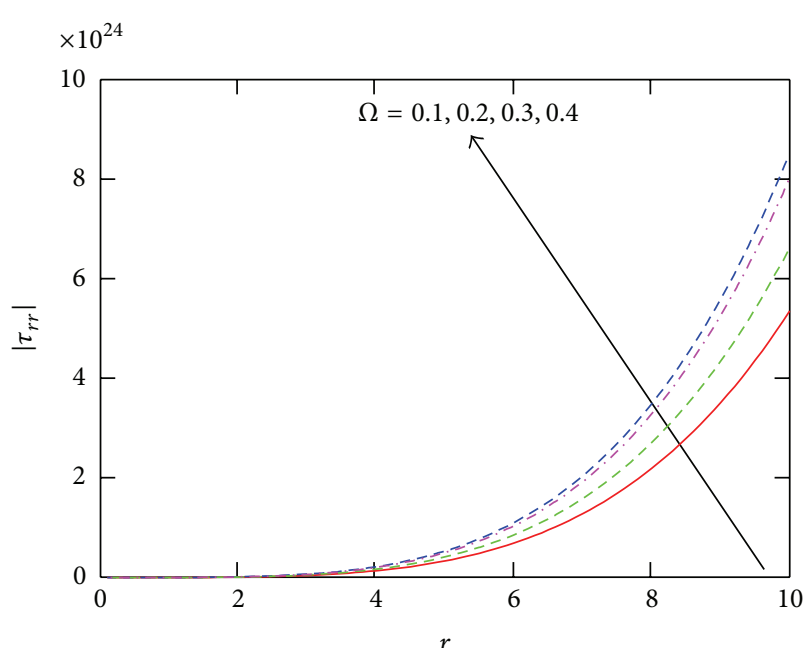

(a)

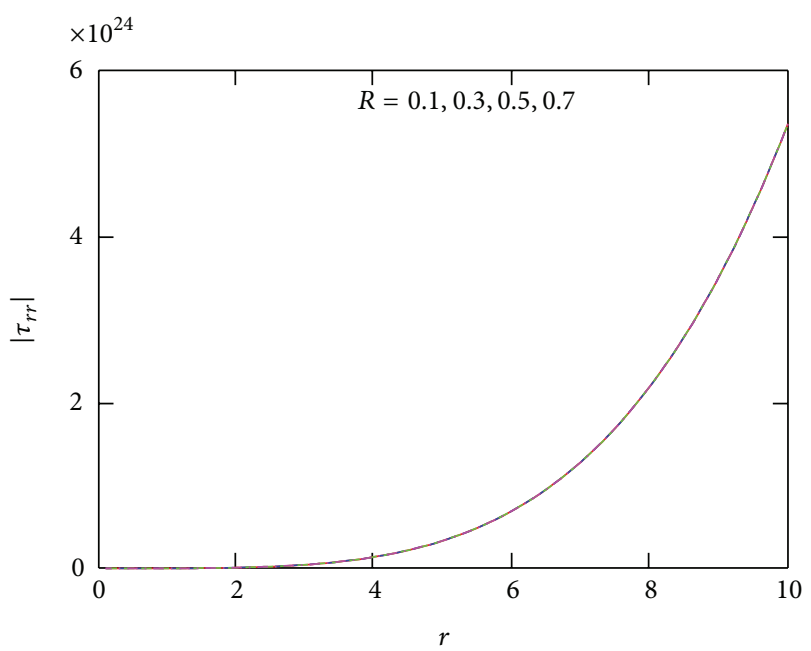

(c)

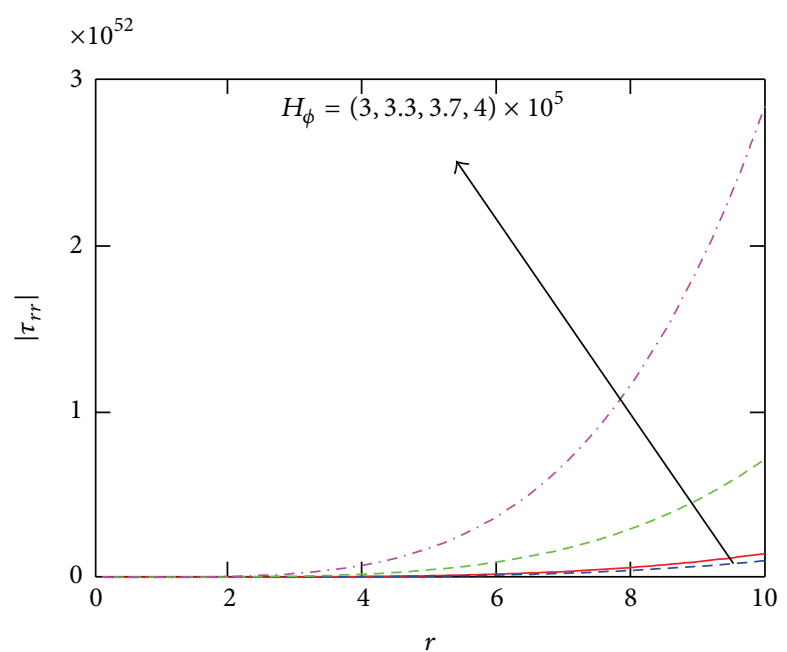

(b)

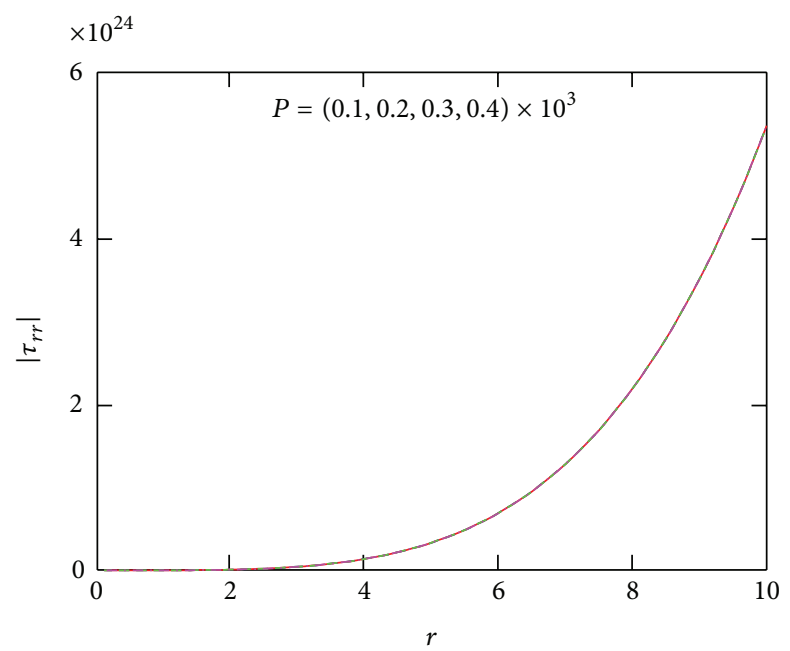

(d)

FIGURE 5: Variation of the absolute values of the radial Maxwell's stress $\tau_{r r}$ with varies values of $\Omega, H_{\phi}, R$, and $P$ with respect to the radius $r$.

$$
\begin{gathered}
\times\left(4 h_{1}^{(2)}\left(m_{2} b\right)\left(\lambda+\mu_{e} H_{\phi}^{2}\right)\right. \\
+b h_{0}^{(2)}\left(m_{2} b\right)\left(\lambda+2 \mu+\mu_{e} H_{\phi}^{2}\right) \\
\left.\left.\left.-b h_{2}^{(2)}\left(m_{2} b\right)\left(\lambda+2 \mu+\mu_{e} H_{\phi}^{2}\right)\right)\right)\right), \\
\Delta_{2}=-2 b h_{0}^{(2)}\left(m_{1} b\right) h_{0}^{(2)}\left(m_{2} a\right) P_{0} \\
\times\left(4 h_{1}^{(2)}\left(m_{1} a\right)\left(\lambda+\mu_{e} H_{\phi}^{2}\right)\right. \\
+b h_{0}^{(2)}\left(m_{1} a\right)\left(\lambda+2 \mu+\mu_{e} H_{\phi}^{2}\right) \\
\left.-b h_{2}^{(2)}\left(m_{2} a\right)\left(\lambda+2 \mu+\mu_{e} H_{\phi}^{2}\right)\right) \\
-h_{0}^{(2)}\left(m_{1} a\right) h_{0}^{(2)}\left(m_{2} b\right) P_{0} \\
\times\left(4 h_{1}^{(2)}\left(m_{1} a\right)\left(\lambda+\mu_{e} H_{\phi}^{2}\right)\right. \\
+b h_{0}^{(2)}\left(m_{1} a\right)\left(\lambda+2 \mu+\mu_{e} H_{\phi}^{2}\right)
\end{gathered}
$$

$$
\begin{gathered}
\left.-b h_{2}^{(2)}\left(m_{1} a\right)\left(\lambda+2 \mu+\mu_{e} H_{\phi}^{2}\right)\right) \\
-(3 \lambda+2 \mu) h_{0}^{(2)}\left(m_{1} a\right) h_{0}^{(2)}\left(m_{1} b\right) T_{0} \alpha_{c} \\
\times\left(4 h_{1}^{(2)}\left(m_{1} a\right)\left(\lambda+\mu_{e} H_{\phi}^{2}\right)\right. \\
+b h_{0}^{(2)}\left(m_{1} a\right)\left(\lambda+2 \mu+\mu_{e} H_{\phi}^{2}\right) \\
-b h_{2}^{(2)}\left(m_{1} a\right)\left(\lambda+2 \mu+\mu_{e} H_{\phi}^{2}\right) \\
+(3 \lambda+2 \mu) h_{0}^{(2)}\left(m_{1} b\right) h_{0}^{(2)}\left(m_{2} a\right) T_{0} \alpha_{c} \\
\times\left(4 h_{1}^{(2)}\left(m_{1} a\right)\left(\lambda+\mu_{e} H_{\phi}^{2}\right)\right. \\
+b h_{0}^{(2)}\left(m_{1} a\right)\left(\lambda+2 \mu+\mu_{e} H_{\phi}^{2}\right) \\
-b h_{2}^{(2)}\left(m_{1} a\right)\left(\lambda+2 \mu+\mu_{e} H_{\phi}^{2}\right) \\
+(3 \lambda+2 \mu) h_{0}^{(2)}\left(m_{1} a\right)^{2} T_{0} \alpha_{c}
\end{gathered}
$$




$$
\begin{aligned}
& \times\left(4 h_{1}^{(2)}\left(m_{1} b\right)\left(\lambda+\mu_{e} H_{\phi}^{2}\right)\right. \\
& +b h_{0}^{(2)}\left(m_{1} b\right)\left(\lambda+2 \mu+\mu_{e} H_{\phi}^{2}\right) \\
& \left.-b h_{2}^{(2)}\left(m_{1} b\right)\left(\lambda+2 \mu+\mu_{e} H_{\phi}^{2}\right)\right) \\
& -(3 \lambda+2 \mu) h_{0}^{(2)}\left(m_{1} a\right) \\
& \times h_{0}^{(2)}\left(m_{2} a\right) T_{0} \alpha_{c} \\
& \times\left(4 h_{1}^{(2)}\left(m_{1} b\right)\left(\lambda+\mu_{e} H_{\phi}^{2}\right)\right. \\
& +b h_{0}^{(2)}\left(m_{1} b\right)\left(\lambda+2 \mu+\mu_{e} H_{\phi}^{2}\right) \\
& \left.\left.\left.-b h_{2}^{(2)}\left(m_{1} b\right)\left(\lambda+2 \mu+\mu_{e} H_{\phi}^{2}\right)\right)\right)\right) \text {, } \\
& \Delta_{3}=\frac{h_{0}^{(2)}\left(m_{2} a\right) T_{0}}{h_{0}^{(2)}\left(m_{1} b\right) h_{0}^{(2)}\left(m_{2} a\right)-h_{0}^{(2)}\left(m_{1} a\right) h_{0}^{(2)}\left(m_{2} b\right)}, \\
& \Delta_{4}=\frac{h_{0}^{(2)}\left(m_{1} a\right) T_{0}}{-h_{0}^{(2)}\left(m_{1} b\right) h_{0}^{(2)}\left(m_{2} a\right)+h_{0}^{(2)}\left(m_{1} a\right) h_{0}^{(2)}\left(m_{2} b\right)} \text {, } \\
& \Delta=-h_{0}^{(2)}\left(m_{1} b\right) h_{0}^{(2)}\left(m_{2} a\right) \\
& \times\left(4 h_{1}^{(2)}\left(m_{1} b\right)\left(\lambda+\mu_{e} H_{\phi}^{2}\right)\right. \\
& +b h_{0}^{(2)}\left(m_{1} b\right)\left(\lambda+2 \mu+\mu_{e} H_{\phi}^{2}\right) \\
& \left.-b h_{2}^{(2)}\left(m_{1} b\right)\left(\lambda+2 \mu+\mu_{e} H_{\phi}^{2}\right)\right) \\
& \times\left(4 h_{1}^{(2)}\left(m_{2} a\right)\left(\lambda+\mu_{e} H_{\phi}^{2}\right)\right. \\
& +b h_{0}^{(2)}\left(m_{2} a\right)\left(\lambda+2 \mu+\mu_{e} H_{\phi}^{2}\right) \\
& \left.-b h_{2}^{(2)}\left(m_{2} a\right)\left(\lambda+2 \mu+\mu_{e} H_{\phi}^{2}\right)\right) \\
& +h_{0}^{(2)}\left(m_{1} a\right) h_{0}^{(2)}\left(m_{2} b\right) \\
& \times\left(4 h_{1}^{(2)}\left(m_{1} b\right)\left(\lambda+\mu_{e} H_{\phi}^{2}\right)\right. \\
& +b h_{0}^{(2)}\left(m_{1} b\right)\left(\lambda+2 \mu+\mu_{e} H_{\phi}^{2}\right) \\
& \left.-b h_{2}^{(2)}\left(m_{1} b\right)\left(\lambda+2 \mu+\mu_{e} H_{\phi}^{2}\right)\right) \\
& \times\left(4 h_{1}^{(2)}\left(m_{2} a\right)\left(\lambda+\mu_{e} H_{\phi}^{2}\right)\right. \\
& +b h_{0}^{(2)}\left(m_{2} a\right)\left(\lambda+2 \mu+\mu_{e} H_{\phi}^{2}\right) \\
& \left.-b h_{2}^{(2)}\left(m_{2} a\right)\left(\lambda+2 \mu+\mu_{e} H_{\phi}^{2}\right)\right) \\
& +h_{0}^{(2)}\left(m_{1} b\right) h_{0}^{(2)}\left(m_{2} a\right) \\
& \times\left(4 h_{1}^{(2)}\left(m_{1} a\right)\left(\lambda+\mu_{e} H_{\phi}^{2}\right)\right. \\
& +b h_{0}^{(2)}\left(m_{1} a\right)\left(\lambda+2 \mu+\mu_{e} H_{\phi}^{2}\right) \\
& \left.-b h_{2}^{(2)}\left(m_{1} a\right)\left(\lambda+2 \mu+\mu_{e} H_{\phi}^{2}\right)\right)
\end{aligned}
$$

$$
\begin{aligned}
& \times( h_{1}^{(2)}\left(m_{2} b\right)\left(\lambda+\mu_{e} H_{\phi}^{2}\right) \\
&+b h_{0}^{(2)}\left(m_{2} b\right)\left(\lambda+2 \mu+\mu_{e} H_{\phi}^{2}\right) \\
&\left.-b h_{2}^{(2)}\left(m_{2} b\right)\left(\lambda+2 \mu+\mu_{e} H_{\phi}^{2}\right)\right) \\
&-h_{0}^{(2)}\left(m_{1} a\right) h_{0}^{(2)}\left(m_{2} b\right) \\
& \times\left(4 h_{1}^{(2)}\left(m_{1} a\right)\left(\lambda+\mu_{e} H_{\phi}^{2}\right)\right. \\
&+b h_{0}^{(2)}\left(m_{1} a\right)\left(\lambda+2 \mu+\mu_{e} H_{\phi}^{2}\right) \\
&\left.-b h_{2}^{(2)}\left(m_{1} a\right)\left(\lambda+2 \mu+\mu_{e} H_{\phi}^{2}\right)\right) \\
& \times\left(4 h_{1}^{(2)}\left(m_{2} b\right)\left(\lambda+\mu_{e} H_{\phi}^{2}\right)\right. \\
&+b h_{0}^{(2)}\left(m_{2} b\right)\left(\lambda+2 \mu+\mu_{e} H_{\phi}^{2}\right) \\
&\left.-b h_{2}^{(2)}\left(m_{2} b\right)\left(\lambda+2 \mu+\mu_{e} H_{\phi}^{2}\right)\right) .
\end{aligned}
$$

Substituting about values of the constants into (4) yields

$$
\begin{aligned}
& u(r, t)=\frac{1}{\Delta}\left[\Delta_{1} h_{1}^{(2)}\left(m_{1} r\right)+\Delta_{2} h_{1}^{(2)}\left(m_{2} r\right)\right] e^{i w t}, \\
& T(r, t)=\left[\Delta_{3} h_{0}^{(2)}\left(m_{1} r\right)+\Delta_{3} h_{0}^{(2)}\left(m_{2} r\right)\right] e^{i w t} .
\end{aligned}
$$

Substitution by $u$ and $T$ into (25)-(27) yields

$$
\begin{aligned}
\sigma_{r r}=\frac{1}{\Delta}([ & {\left[\left(\mu+\frac{\lambda}{2}\right) h_{0}^{(2)}\left(m_{1} r\right)-\left(\mu+\frac{\lambda}{2}\right) h_{2}^{(2)}\left(m_{1} r\right)\right] \Delta_{1} } \\
& +\left(\mu+\frac{\lambda}{2}\right)\left[h_{0}^{(2)}\left(m_{2} r\right)-h_{2}^{(2)}\left(m_{2} r\right)\right] \Delta_{2} \\
& \left.+\frac{2 \lambda}{r} h_{1}^{(2)}\left(m_{1} r\right) \Delta_{1}+\frac{2 \lambda}{r} h_{1}^{(2)}\left(m_{2} r\right) \Delta_{2}\right) \\
& \left.-(3 \lambda+2 \mu) \alpha_{c}\left[\Delta_{3} h_{0}^{(2)}\left(m_{1} r\right)+\Delta_{4} h_{0}^{(2)}\left(m_{2} r\right)\right]\right] e^{i w t} \\
\sigma_{\phi \phi}=\sigma_{\theta \theta}= & \frac{1}{\Delta}(\lambda+\mu)\left[\Delta_{1} h_{1}^{(2)}\left(m_{1} r\right)+\Delta_{2} h_{1}^{(2)}\left(m_{2} r\right)\right] \\
& +\lambda\left[\frac{\Delta_{1}}{2}\left(h_{0}^{(2)}\left(m_{1} r\right)-h_{2}^{(2)}\left(m_{1} r\right)\right)\right. \\
& \left.+\frac{\Delta_{2}}{2}\left(h_{0}^{(2)}\left(m_{2} r\right)-h_{2}^{(2)}\left(m_{2} r\right)\right)\right] \\
& \quad(3 \lambda+2 \mu) \alpha_{c} \\
& \left.\times\left[\Delta_{3} h_{0}^{(2)}\left(m_{1} r\right)+\Delta_{4} h_{0}^{(2)}\left(m_{2} r\right)\right]\right) e^{i w t},
\end{aligned}
$$




$$
\begin{aligned}
\tau_{r r}=\frac{1}{\Delta}\left(\mu_{e} H_{\phi}^{2}[(\right. & \frac{1}{2}\left[h_{0}^{(2)}\left(m_{1} r\right)-h_{2}^{(2)}\left(m_{1} r\right)\right] \\
& \left.+\frac{2}{r} h_{1}^{(2)}\left(m_{1} r\right)\right) \Delta_{1} \\
& +\frac{1}{2}\left[\left(h_{0}^{(2)}\left(m_{2} r\right)-h_{2}^{(2)}\left(m_{2} r\right)\right)\right. \\
& \left.\left.\left.+\frac{2}{r} h_{1}^{(2)}\left(m_{2} r\right)\right] \Delta_{2}\right] e^{i w t}\right) .
\end{aligned}
$$

\section{Numerical Results and Discussion}

In order to illustrate the theoretical results obtained in the preceding section and to compare various theories of thermoelasticity formulated earlier, we present some numerical results for the physical constants. For the purposes of numerical calculations, the copper material was chosen. The constants of the problem given by Sokolnikoff [33] and Thomas [34] are

$$
\begin{aligned}
& K=0.0921 \times 10^{13} \mathrm{Wm}^{-1} \mathrm{~K}^{-1}, \quad \alpha_{t}=1.28 \times 10^{-9} \mathrm{~K}^{-1}, \\
& c_{e}=787 \times 10^{3} \mathrm{JKg}^{-1} \mathrm{~K}^{-1}, \\
& \rho=2660 \mathrm{Kgm}^{-3}, \quad \lambda=5.65 \times 10^{10} \mathrm{Nm}^{-2}, \\
& \mu=2.46 \times 10^{10} \mathrm{Nm}^{-2}, \\
& \eta=8886.73 \mathrm{sm}^{-2}, \quad T_{0}=293 \mathrm{~K}, \quad a=0.5, \\
& \beta=220.9 \times 10^{9}, \quad \omega=0.2 .
\end{aligned}
$$

The values of the absolute radial displacement $|u|$, absolute temperature distribution $|T|$, absolute stresses $\left|\sigma_{r r}\right|,\left|\sigma_{\theta \theta}\right|$, and absolute value of the radial Maxwell's stress $\left|\tau_{r r}\right|$. The output is plotted in Figures 1-5. Figure 1 shows the value of radial displacement $|u|$ with respect to $r$ for different values of the rotation $\Omega$, magnetic field $H_{\phi}, R$, and initial stress $P$. These figures indicate that the medium along $r$ undergoes expansion deformation due to the thermal shock, while the other shows the tension deformation. The effect of rotation $\Omega$ and magnetic field $H_{\phi}$ on radial displacement becomes large that increases and decreases with increasing of the rotation and magnetic field, respectively, while there is no effect of $R$ and initial stress. At a given instant, the radial displacement is finite, which is due to the effect of rotation, magnetic field, $r$ and initial stress.

Figure 2 shows that the value of temperature distribution $T$ with respect to $r$, while the solution is notably different inside the sphere. This is due to the fact that the thermal waves in the coupled theory travel with an infinite speed. The effects of rotation $\Omega$, magnetic field $H_{\phi}, R$, and initial stress $P$ on temperature distribution have been shown in Figure 2 . The temperature increases with increasing of the rotation and magnetic field, while there is no effect of the $R$ and initial stress $P$.
Figures 3 and 4 show the variations of absolute radial stress $\left|\sigma_{r r}\right|$ and absolute tangential stress $\left|\sigma_{\phi \phi}\right|$ with respect to the radius $r$, respectively. The values of radial stress and tangential stress are increased with increasing of rotation and magnetic field due to the effect of rotation and magnetic field for all values of the radius $r$, while there is no effect of $R$ and initial stress. For the values of $\left|\sigma_{r r}\right|$ and $\left|\sigma_{\phi \phi}\right|$, depicting the effect of rotation and magnetic field. It is shown that the radial stress is tension in its nature.

Figure 5 shows that the values of absolute values of the radial Maxwell's stress $\left|\tau_{r r}\right|$ with respect to $r$. The effects of rotation $\Omega$, magnetic field $H_{\phi}, R$, and initial stress $P$ on radial Maxwell's stress which it increases with increasing of rotation and magnetic field, while there is no effect of $R$ and initial stress.

\section{Conclusion}

Due to the complicated nature of the governing equations of the magnetothermoelastic theory, the done works in this field are unfortunately limited. The method used in this study provides a quite successful in dealing with such problems. This method gives exact solutions in the elastic medium without any restrictions on the actual physical quantities that appear in the governing equations of the considered problem. Important phenomena are observed in these computations.

(i) It was found that large values of rotation and magnetic field give close results. The case is quite different when we consider small value of rotation. The coupled theory predicts infinite speeds of wave propagation. The solutions are obtained in the context of thermoelasticity theory.

(ii) Comparing Figures 1-5 for thermoelastic medium, it was found that $u, \sigma_{r r}, \sigma_{\phi \phi}$, and $\tau_{r r}$ have the same behavior in both media. But with the passage of magnetic field and rotation, the numerical values of $u, \sigma_{r r}, \sigma_{\phi \phi}$, and $\tau_{r r}$ in thermoelastic medium are large due to the influences of magnetic field, rotation, $R$, and initial stress $P$.

(iii) The results presented in this paper will be very helpful for researchers concerned with material science, designers of new materials, low-temperature physicists, and for those working on the development of a theory of hyperbolic propagation of hyperbolic thermodiffusion. Study of the phenomenon of rotation, magnetic field, $R$, and initial stress is also used to improve the conditions of oil extractions.

\section{Conflict of Interests}

The authors declare that they have no conflict of interests regarding the publication of this paper. 


\section{Acknowledgment}

The authors extend their gratitude to Professor A. M. AbdAlla of the Faculty of Science, Taif University, Saudi Arabia, for his help in revising this work.

\section{References}

[1] W. Nowacki, "Dynamical problems of thermodiffusion in solids I," Bulletin de l'Académie Polonaise des Sciences, vol. 22, no. 1, pp. 55-64, 1974.

[2] W. Nowacki, "Dynamical problems of thermodiffusion in solids II," Bulletin de l'Académie Polonaise des Sciences, vol. 22, no. 1, pp. 129-135, 1974.

[3] W. Nowacki, "Dynamical problems of thermo Diffusion in solid III," Bulletin de l'Académie Polonaise des Sciences, vol. 22, no. 1, pp. 257-266, 1974.

[4] H. W. Lord and Y. Shulman, "A generalized dynamical theory of thermoelasticity," Journal of the Mechanics and Physics of Solids, vol. 7, no. 5, pp. 71-75, 1967.

[5] R. S. Dhaliwal and H. H. Sherief, "Generalized thermoelasticity for an Isotropic media," Quarterly of Applied Mathematics, vol. 33, no. 1, pp. 1-8, 1980.

[6] A. M. Abd-Alla and S. R. Mahmoud, "Magneto-thermoelastic problem in rotating non-homogeneous orthotropic hollow cylinder under the hyperbolic heat conduction model," Meccanica, vol. 45, no. 4, pp. 451-462, 2010.

[7] A. M. Abd-Alla, A. N. Abd-Alla, and N. A. Zeidan, "Thermal stresses in a nonhomogeneous orthotropic elastic multilayered cylinder," Journal of Thermal Stresses, vol. 23, no. 5, pp. 413-428, 2000.

[8] M. A. Ezzat and H. M. Youssef, "Generalized magnetothermoelasticity in a perfectly conducting medium," International Journal of Solids and Structures, vol. 42, no. 24-25, pp. 6319-6334, 2005.

[9] M. I. A. Othman, "Effect of rotation and relaxation time on a thermal shock problem for a half-space in generalized thermoviscoelasticity," Acta Mechanica, vol. 174, no. 3-4, pp. 129-143, 2005.

[10] M. I. A. Othman, "Lord-Shulman theory under the dependence of the modulus of elasticity on the reference temperature in twodimensional generalized thermoelasticity," Journal of Thermal Stresses, vol. 25, no. 11, pp. 1027-1045, 2002.

[11] A. E. Green and K. A. Lindsay, "Thermoelasticity," Journal of Elasticity, vol. 2, no. 1, pp. 1-7, 1972.

[12] M. I. A. Othman and Y. Song, "Effect of rotation on plane waves of generalized electro-magneto-thermoviscoelasticity with two relaxation times," Applied Mathematical Modelling, vol. 32, no. 5, pp. 811-825, 2008.

[13] M. I. A. Othman and Y. Song, "Reflection of magneto-thermoelastic waves from a rotating elastic half-space," International Journal of Engineering Science, vol. 46, no. 5, pp. 459-474, 2008.

[14] H. H. Sherief, F. A. Hamza, and H. A. Saleh, "The theory of generalized thermoelastic diffusion," International Journal of Engineering Science, vol. 42, no. 5-6, pp. 591-608, 2004.

[15] H. H. Sherief and H. A. Saleh, "A half-space problem in the theory of generalized thermoelastic diffusion," International Journal of Solids and Structures, vol. 42, no. 15, pp. 4484-4494, 2005.

[16] B. Singh, "Reflection of SV waves from the free surface of an elastic solid in generalized thermoelastic diffusion," Journal of Sound and Vibration, vol. 291, no. 3-5, pp. 764-778, 2006.
[17] R. Kumar and T. Kansal, "Propagation of Lamb waves in transversely isotropic thermoelastic diffusive plate," International Journal of Solids and Structures, vol. 45, no. 3-4, pp. 5890-5913, 2008.

[18] P. Ram, N. Sharma, and R. Kumar, “Thermomechanical response of generalized thermoelastic diffusion with one relaxation time due to time harmonic sources," International Journal of Thermal Sciences, vol. 47, no. 3, pp. 315-323, 2008.

[19] M. Aouadi, "A problem for an infinite elastic body with a spherical cavity in the theory of generalized thermoelastic diffusion," International Journal of Solids and Structures, vol. 44, no. 17, pp. 5711-5722, 2007.

[20] S. M. Abo-Dahab and B. Singh, "Influences of magnetic field on wave propagation in generalized thermoelastic solid with diffusion," Archives of Mechanics, vol. 61, no. 2, pp. 121-136, 2009.

[21] M. I. A. Othman, S. Y. Atwa, and R. M. Farouk, "The effect of diffusion on two-dimensional problem of generalized thermoelasticity with Green-Naghdi theory," International Communications in Heat and Mass Transfer, vol. 36, no. 8, pp. 857-864, 2009.

[22] R.-H. Xia, X.-G. Tian, and Y.-P. Shen, "The influence of diffusion on generalized thermoelastic problems of infinite body with a cylindrical cavity," International Journal of Engineering Science, vol. 47, no. 5-6, pp. 669-679, 2009.

[23] S. Deswal and K. Kalkal, "A two-dimensional generalized electro-magneto-thermoviscoelastic problem for a half-space with diffusion," International Journal of Thermal Sciences, vol. 50, no. 5, pp. 749-759, 2011.

[24] A. M. Abd-Alla and S. M. Abo-Dahab, "Time-harmonic sources in a generalized magneto-thermo-viscoelastic continuum with and without energy dissipation," Applied Mathematical Modelling, vol. 33, no. 5, pp. 2388-2402, 2009.

[25] S. M. Abo-Dahab and R. A. Mohamed, "Influence of magnetic field and hydrostatic initial stress on wave reflection from a generalized thermoelastic solid half-space," Journal of Vibration and Control, vol. 16, no. 5, pp. 685-699, 2010.

[26] A. M. Abd-Alla, S. M. Abo-Dahab, H. A. Hammad, and S. R. Mahmoud, "On generalized magneto-thermoelastic rayleigh waves in a granular medium under the influence of a gravity field and initial stress," Journal of Vibration and Control, vol. 17, no. 1, pp. 115-128, 2011.

[27] S. K. Roychoudhuri and S. Mukhopadhyay, "Effect of rotation and relaxation times on plane waves in generalized thermo-visco-elasticity," International Journal of Mathematics and Mathematical Sciences, vol. 23, no. 7, pp. 497-505, 2000.

[28] S. M. Abo-Dahab, "Propagation of $P$ waves from stress-free surface elastic half-space with voids under thermal relaxation and magnetic field," Applied Mathematical Modelling, vol. 34, no. 7, pp. 1798-1806, 2010.

[29] A. M. Abd-Alla, S. R. Mahmoud, S. M. Abo-Dahab, and M. I. Helmy, "Propagation of $S$-wave in a non-homogeneous anisotropic incompressible and initially stressed medium under influence of gravity field," Applied Mathematics and Computation, vol. 217, no. 9, pp. 4321-4332, 2011.

[30] A. M. Abd-Alla, S. M. Abo-Dahab, and S. R. Mahmoud, "Wave propagation modeling in cylindrical human long wet bones with cavity," Meccanica, vol. 46, no. 6, pp. 1413-1428, 2011.

[31] S. M. Ahmed and S. M. Abo-Dahab, "Propagation of Love waves in an orthotropic granular layer under initial stress overlying a semi-infinite granular medium," Journal of Vibration and Control, vol. 16, no. 12, pp. 1845-1858, 2010.

[32] J. D. Kraus, Electromagnetics, McGraw-Hill, New York, NY, USA, 1984. 
[33] I. S. Sokolnikoff, Mathematical Theory of Elasticity, Dover, New York, NY, USA, 1946.

[34] L. Thomas, Fundamentals of Heat Transfer, Prentice Hall, Englewood Cliffs, NJ, USA, 1980. 


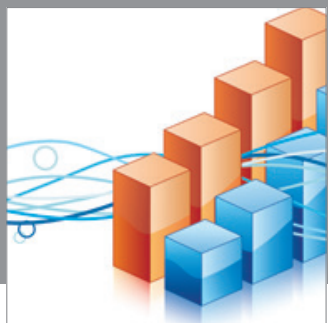

Advances in

Operations Research

mansans

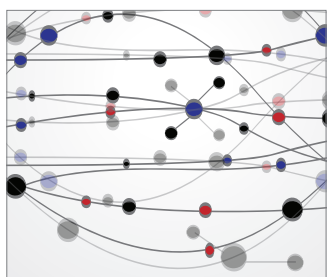

The Scientific World Journal
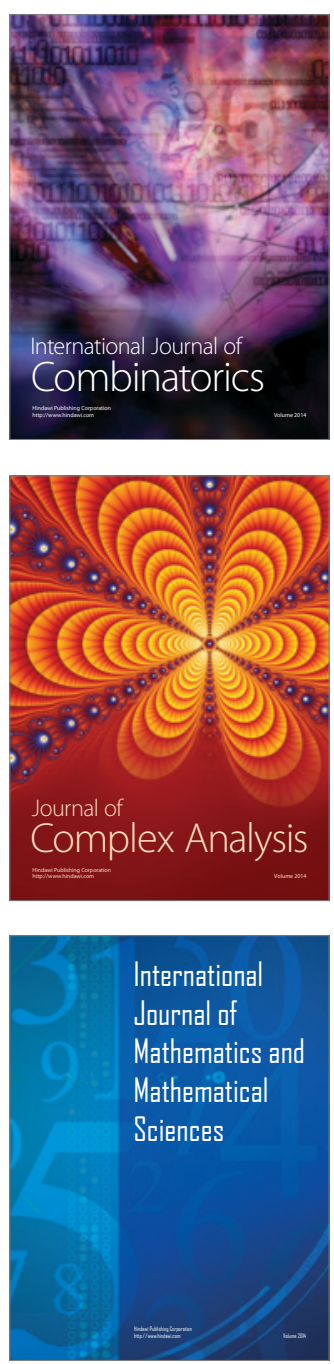
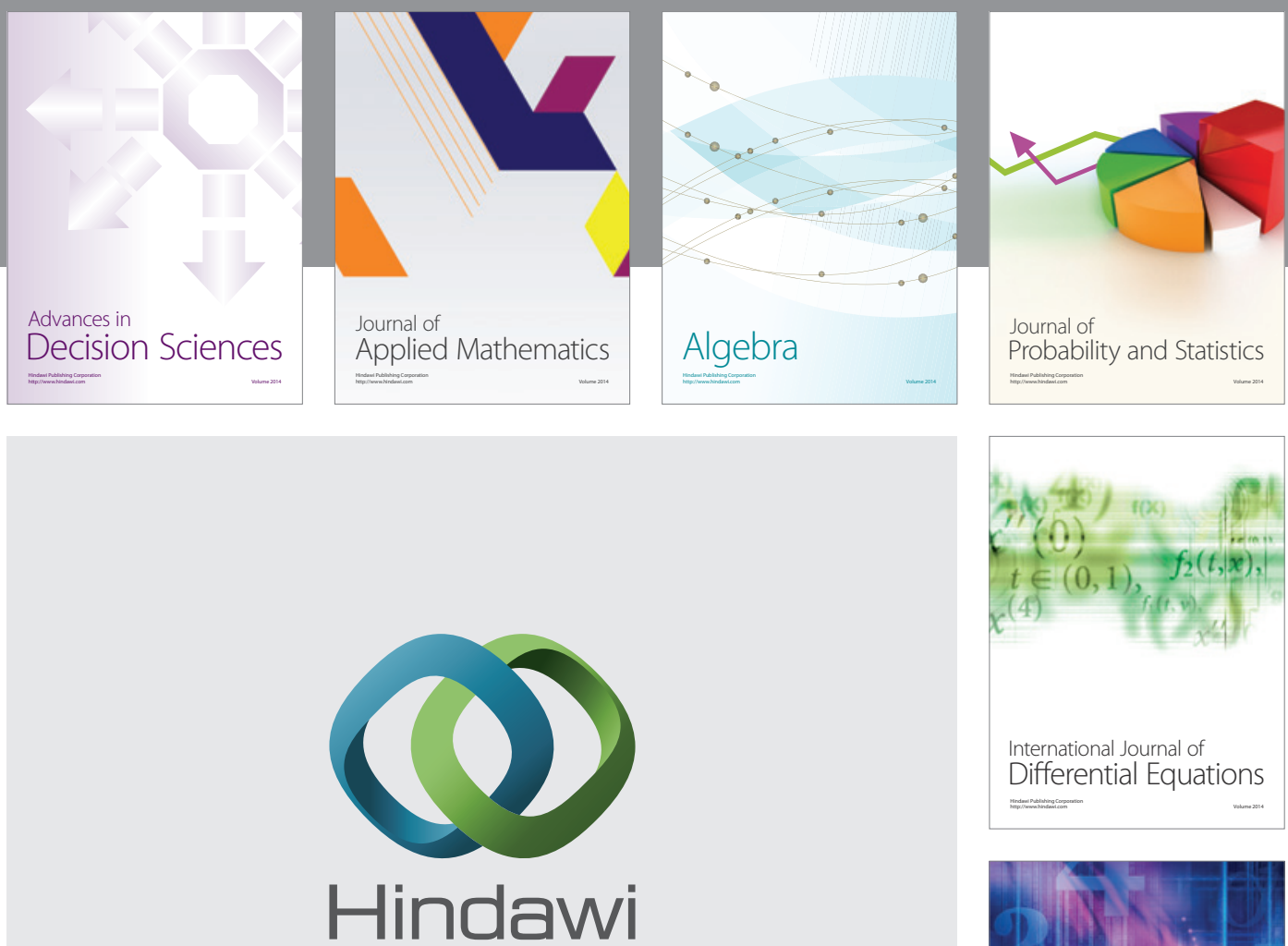

Submit your manuscripts at http://www.hindawi.com
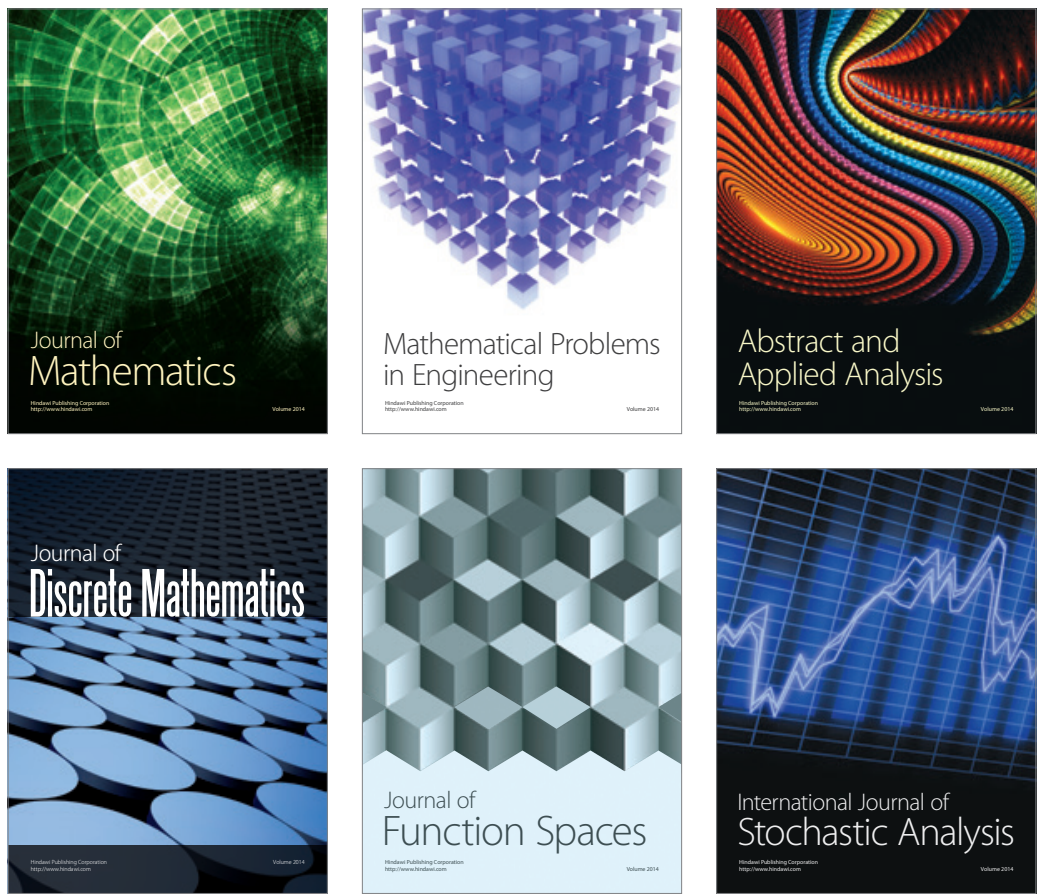

Journal of

Function Spaces

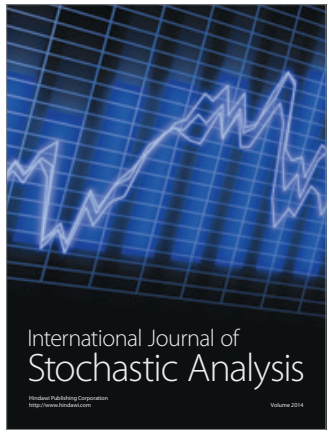

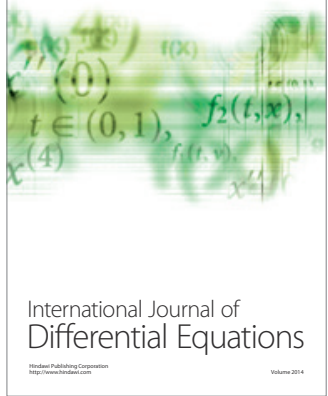
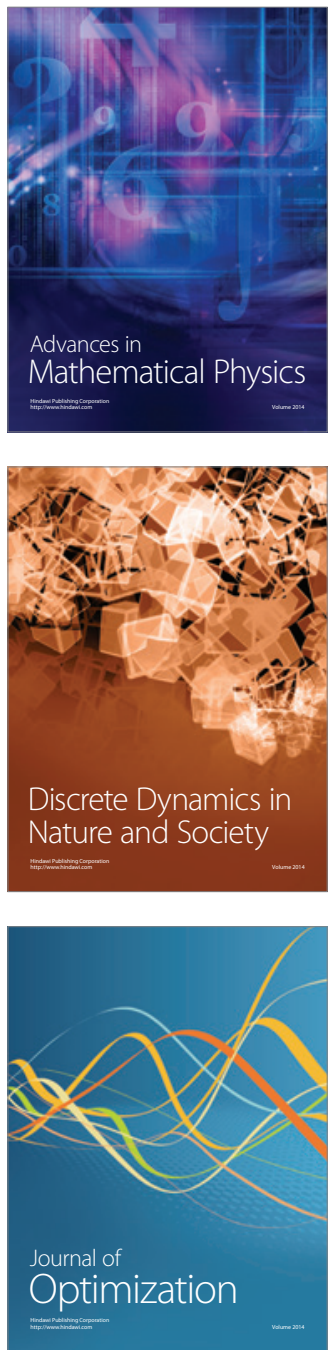